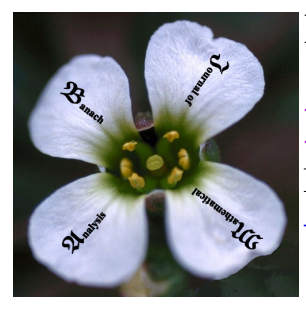

Banach J. Math. Anal. 8 (2014), no. 1, 148-178

Banach Journal of $\mathbf{M}_{\text {athematical }} \mathbf{A}_{\text {nalysis }}$

ISSN: $1735-8787$ (electronic)

www.emis.de/journals/BJMA/

\title{
SOME OPTIMIZATION PROBLEMS ON RANKS AND INERTIAS OF MATRIX-VALUED FUNCTIONS SUBJECT TO LINEAR MATRIX EQUATION RESTRICTIONS
}

\author{
YONGGE TIAN
}

Communicated by F. Zhang

\begin{abstract}
Matrix rank and inertia optimization problems are a class of discontinuous optimization problems in which the decision variables are matrices running over certain matrix sets, while the ranks and inertias of the variable matrices are taken as integer-valued objective functions. In this paper, we establish a group of explicit formulas for calculating the maximal and minimal values of the rank and inertia objective functions of the Hermitian matrix-valued function $A_{1}-B_{1} X B_{1}^{*}$ subject to the common Hermitian solution of a pair of consistent matrix equations $B_{2} X B_{2}^{*}=A_{2}$ and $B_{3} X B_{3}^{*}=A_{3}$, and Hermitian solution of the consistent matrix equation $B_{4} X=A_{4}$, respectively. Many consequences are obtained, in particular, necessary and sufficient conditions are established for the triple matrix equations $B_{1} X B_{1}^{*}=A_{1}$, $B_{2} X B_{2}^{*}=A_{2}$ and $B_{3} X B_{3}^{*}=A_{3}$ to have a common Hermitian solution, as well as necessary and sufficient conditions for the two matrix equations $B_{1} X B_{1}^{*}=A_{1}$ and $B_{4} X=A_{4}$ to have a common Hermitian solution.
\end{abstract}

\section{INTRODUCTION}

The matrix approximation problem is to approximate optimally, with respect to some criteria, a matrix by one of the same dimension from a given feasible matrix set. Assume that $A$ is a matrix to be approximated. Then a conventional statement of general matrix optimization problems of $A$ from this point of view

Date: Received: 13 March 2013; Accepted: 30 May 2013.

2010 Mathematics Subject Classification. Primary 15A24; Secondary 15B57, 49K30, 65K10, 90C11, 90C22, 47A62.

Key words and phrases. Matrix-valued function, matrix equation, rank, inertia, optimization. 
can be written as

$$
\text { minimize } \rho(A-X) \text { subject to } X \in \mathcal{S},
$$

where $\rho(\cdot)$ is certain objective function, which is usually taken as the determinant, trace, norms, rank, inertia of matrix, and $\mathcal{S}$ is a given feasible matrix set. A bestknown case of (1.1) is to minimize the norm $\|A-X\|_{F}^{2}$ subject to $X \in \mathcal{S}$.

In this paper, we take two matrix sets as

$$
\begin{aligned}
& \mathcal{S}=\left\{X \in \mathbb{C}_{\mathrm{H}}^{n} \mid\left[B_{2} X B_{2}^{*}, B_{3} X B_{3}^{*}\right]=\left[A_{2}, A_{3}\right]\right\}, \\
& \mathcal{T}=\left\{X \in \mathbb{C}_{\mathrm{H}}^{n} \mid B_{4} X=A_{4}\right\},
\end{aligned}
$$

where $A_{i} \in \mathbb{C}_{\mathrm{H}}^{m_{i}}, B_{i} \in \mathbb{C}^{m_{i} \times n}, A_{4}, B_{4} \in \mathbb{C}^{m_{4} \times n}$ are given, $i=2,3$, and $X \in \mathbb{C}_{\mathrm{H}}^{n}$ is a variable matrix, and study the following constrained optimization problems.

Problem 1.1. For the Hermitian matrix-valued function $A_{1}-B_{1} X B_{1}^{*}$, where $A_{1} \in \mathbb{C}_{\mathrm{H}}^{m_{1}}$ and $B_{1} \in \mathbb{C}^{m_{1} \times n}$, and the matrix set in $\mathcal{S}$ in (1.2), establish explicit formulas for calculating

$$
\begin{aligned}
& \max _{X \in \mathcal{S}} r\left(A_{1}-B_{1} X B_{1}^{*}\right), \\
& \min _{X \in \mathcal{S}} r\left(A_{1}-B_{1} X B_{1}^{*}\right), \\
& \min _{X \in \mathcal{S}} i_{ \pm}\left(A_{1}-B_{1} X B_{1}^{*}\right), \\
& \min _{X \in \mathcal{S}} i_{ \pm}\left(A_{1}-B_{1} X B_{1}^{*}\right),
\end{aligned}
$$

Problem 1.2. Establish necessary and sufficient conditions for the following three linear Hermitian matrix equations

$$
\left[B_{1} X B_{1}^{*}, B_{2} X B_{2}^{*}, B_{3} X B_{3}^{*}\right]=\left[A_{1}, A_{2}, A_{3}\right]
$$

to have a common Hermitian solution, and establish necessary and sufficient conditions for the Löwner partial ordering matrix inequalities

$$
A_{1}-B_{1} X B_{1}^{*}>0, A_{1}-B_{1} X B_{1}^{*} \geqslant 0, A_{1}-B_{1} X B_{1}^{*}<0, A_{1}-B_{1} X B_{1}^{*} \leqslant 0
$$

to hold respectively for an (all) $X \in \mathcal{S}$ in (1.2).

Problem 1.3. For the Hermitian matrix-valued function $A_{1}-B_{1} X B_{1}^{*}$, where $A_{1} \in \mathbb{C}_{\mathrm{H}}^{m_{1}}$ and $B_{1} \in \mathbb{C}^{m_{1} \times n}$, and the matrix set $\mathcal{T}$ in (1.3), establish explicit formulas for calculating

$$
\begin{aligned}
& \max _{X \in \mathcal{T}} r\left(A_{1}-B_{1} X B_{1}^{*}\right), \\
& \min _{X \in \mathcal{T}} r\left(A_{1}-B_{1} X B_{1}^{*}\right), \\
& \max _{X \in \mathcal{T}} i_{ \pm}\left(A_{1}-B_{1} X B_{1}^{*}\right), \\
& \min _{X \in \mathcal{T}} i_{ \pm}\left(A_{1}-B_{1} X B_{1}^{*}\right) .
\end{aligned}
$$

Problem 1.4. Establish necessary and sufficient conditions for the following two matrix equations

$$
\left[B_{1} X B_{1}^{*}, B_{4} X\right]=\left[A_{1}, A_{4}\right]
$$


to have a common Hermitian solution and positive semi-definite solution, respectively, and establish necessary and sufficient conditions for the Löwner partial ordering matrix inequalities

$A_{1}-B_{1} X B_{1}^{*}>0, A_{1}-B_{1} X B_{1}^{*} \geqslant 0, A_{1}-B_{1} X B_{1}^{*}<0, A_{1}-B_{1} X B_{1}^{*} \leqslant 0$

to hold, respectively, for an (all) $X \in \mathcal{T}$ in (1.3).

Throughout this paper,

$\mathbb{C}^{m \times n}$ and $\mathbb{C}_{\mathrm{H}}^{m}$ stand for the sets of all $m \times n$ complex matrices and $m \times m$ complex Hermitian matrices; respectively;

$A^{T}, A^{*}, r(A), \mathscr{R}(A)$ stand for the transpose, conjugate transpose, rank and range (column space) of a matrix $A \in \mathbb{C}^{m \times n}$, respectively;

$I_{m}$ denotes the identity matrix of order $m$;

$[A, B]$ denotes a row block matrix consisting of $A$ and $B$;

$A>0(A \geqslant 0)$ means that $A$ is Hermitian positive definite (Hermitian positive semi-definite);

two $A, B \in \mathbb{C}_{\mathrm{H}}^{m}$ are said to satisfy the inequality $A>B(A \geqslant B)$ in the Löwner partial ordering if $A-B$ is positive definite (positive semi-definite); the Moore-Penrose inverse of $A \in \mathbb{C}^{m \times n}$, denoted by $A^{\dagger}$, is defined to be the unique solution $X$ satisfying the four matrix equations $A X A=A$, $X A X=X,(A X)^{*}=A X$ and $(X A)^{*}=X A$, which satisfies $A A^{\dagger}=A^{\dagger} A$ if $A=A^{*}$;

a matrix $X$ is called a Hermitian $g$-inverse of $A \in \mathbb{C}_{\mathrm{H}}^{m}$, denoted by $A^{-}$, if it satisfies both $A X A=A$ and $X=X^{*}$;

$E_{A}$ and $F_{A}$ stand for $E_{A}=I_{m}-A A^{\dagger}$ and $F_{A}=I_{n}-A^{\dagger} A$, and the ranks of $E_{A}$ and $F_{A}$ are given by $r\left(E_{A}\right)=m-r(A)$ and $r\left(F_{A}\right)=n-r(A)$;

$i_{+}(A)$ and $i_{-}(A)$, usually called the partial inertia of $A \in \mathbb{C}_{\mathrm{H}}^{m}$, are defined to be the numbers of the positive and negative eigenvalues of $A$ counted with multiplicities, respectively, which satisfy $r(A)=i_{+}(A)+i_{-}(A)$.

Once close-form formulas for the extremal ranks and inertias of a matrix-valued function are established, they can directly be used to describe some behaviors of the matrix-valued function, for example,

(I) the maximal and minimal dimensions of the row and column spaces of the matrix-valued function;

(II) nonsingularity of the matrix-valued function when it is square;

(III) solvability of the corresponding matrix equation;

(IV) rank, inertia and range invariance of the matrix-valued function;

(V) semi-definiteness of the matrix-valued function, etc.

On the other hand, matrix rank and inertia optimization problems are NP-hard in general due to the discontinuity and combinational nature of rank and inertia of a matrix and the complexity of algebraic structure of the given matrix set $\mathcal{S}$.

Mappings between matrix spaces with symmetric patterns can be constructed arbitrarily, but the matrix-valued function $\phi(X)=A-B X B^{*}$ is the simplest case among all matrix-valued functions with symmetric patterns. This function is the starting point in dealing with various complicated matrix-valued functions with symmetric patterns. In recent years, the present author and his coauthors gave 
a comprehensive study on the $\phi(X)$ and its algebraic properties, and obtained many fundamental results. The work done includes:

(i) establishing expansion formulas for calculating the (global extremal) rank and inertia of $\phi(X)$ when $X$ running over $\mathbb{C}_{\mathrm{H}}^{n}$, and the matrix $X$ such that the objective rank and inertia functions attain the global extremal ranks and inertias, see [17, 27, 39];

(ii) characterizing nonsingularity, positive definiteness, positive semi-definiteness, rank and inertia invariance, etc., of $\phi(X)$, see [27, 39];

(iii) establishing canonical forms of $\phi(X)$ under generalized singular value decompositions and characterizing their algebraic properties, see [17];

(iv) deriving solutions and least-squares solutions of the matrix equation $\phi(X)=$ 0 and characterizing their algebraic properties, see [14, 18, 31, 33, 36];

(v) deriving solutions of the matrix inequalities $\phi(X)>0(\geqslant 0,<0, \leqslant 0)$ and characterizing their properties, see [27];

(vi) minimizing $\operatorname{tr}\left[\phi(X) \phi^{*}(X)\right]$ s.t. $r[\phi(X)]=\min$, see [36];

(vii) establishing formulas for calculating the extremal ranks and inertias of $\phi(X)$ under the restrictions $r(X) \leqslant k$ and/or $\pm X \geqslant 0$, see [32];

(viii) establishing formulas for calculating the extremal ranks and inertias of $\phi(X)$ subject to a consistent matrix equation $C X C^{*}=D$, see [16].

This seminal work was also extended to some general matrix-valued functions with symmetric patterns, such as,

$$
A-B X-(B X)^{*}, \quad A-B X B^{*}-C Y C^{*}, \quad A-B X C-(B X C)^{*},
$$

where $X$ and $Y$ are (Hermitian) variable matrices of appropriate sizes, see, e.g., $[13,14,15,16,29,31,33]$.

We shall use some pure algebraic operations on matrices to derive two groups of closed-form formulas for calculating the global extremal values of the objective functions in (1.4)-(1.7) and (1.10)-(1.13), and then to present a variety of valuable consequences of these formulas.

Since variable entries in a matrix-valued function are often regarded as continuous variables in some feasible sets, while the objective functions - the rank and inertia of the matrix-valued function take values only from a finite set of nonnegative integers. Hence, (1.4)-(1.7) and (1.10)-(1.13) can be regarded as continuous-integer optimization problems subject to equality constraints. This kind of non-smooth optimization problems cannot be solved by using various optimization methods for solving continuous or discrete cases. There is no rigorous mathematical theory for solving a general rank and inertia optimization problem due to the discontinuity and nonconvexity of rank and inertia of matrix. In fact, it has been realized that rank and inertia optimization problems have deep connections with computational complexity, and are regarded as NP-hard in general; see, e.g., [1, 2, 3, 4, 6, 7, 8, 10, 11, 20, 21, 22, 23, 25]. Fortunately, some special rank and inertia optimization problems now can be solved analytically by pure algebraical methods. 


\section{PRELiminaries}

The following are some known results for ranks and inertias of matrices and their usefulness, which will be used in Sections 3 and 4 .

Lemma 2.1 ([27]). Let $\mathcal{H}$ be a matrix set in $\mathbb{C}_{\mathrm{H}}^{m}$. Then, the following hold.

(a) $\mathcal{H}$ has a matrix $X>0(X<0)$ if and only if

$$
\max _{X \in \mathcal{H}} i_{+}(X)=m \quad\left(\max _{X \in \mathcal{H}} i_{-}(X)=m\right)
$$

(b) All $X \in \mathcal{H}$ satisfy $X>0(X<0)$, namely, $\mathcal{H}$ is a subset of the cone of positive definite matrices (negative definite matrices), if and only if

$$
\min _{X \in \mathcal{H}} i_{+}(X)=m \quad\left(\min _{X \in \mathcal{H}} i_{-}(X)=m\right)
$$

(c) $\mathcal{H}$ has a matrix $X \geqslant 0(X \leqslant 0)$ if and only if

$$
\min _{X \in \mathcal{H}} i_{-}(X)=0 \quad\left(\min _{X \in \mathcal{H}} i_{+}(X)=0\right) .
$$

(d) All $X \in \mathcal{H}$ satisfy $X \geqslant 0(X \leqslant 0)$ namely, $\mathcal{H}$ is a subset of the cone of positive semi-definite matrices (negative semi-definite matrices), if and only if

$$
\max _{X \in \mathcal{H}} i_{-}(X)=0 \quad\left(\max _{X \in \mathcal{H}} i_{+}(X)=0\right) .
$$

The question of whether a given function is definite or semi-definite everywhere is ubiquitous in mathematics and applications. Lemma 2.1(a)-(d) show that if some explicit formulas for calculating the global maximal and minimal inertias of a given Hermitian matrix-valued function are established, we can use them, as demonstrated in sections below, to derive necessary and sufficient conditions for the Hermitian matrix-valued function to be definite or semi-definite.

Lemma 2.2 ([19]). Let $A \in \mathbb{C}^{m \times n}, B \in \mathbb{C}^{m \times p}$ and $C \in \mathbb{C}^{q \times n}$. Then, the following rank expansion formulas hold

$$
\begin{aligned}
r[A, B] & =r(A)+r\left(E_{A} B\right)=r(B)+r\left(E_{B} A\right), \\
r\left[\begin{array}{l}
A \\
C
\end{array}\right] & =r(A)+r\left(C F_{A}\right)=r(C)+r\left(A F_{C}\right), \\
r\left[\begin{array}{cc}
A & B \\
C & 0
\end{array}\right] & =r(B)+r(C)+r\left(E_{B} A F_{C}\right) .
\end{aligned}
$$


Three useful rank expansion formulas derived from (2.3) are

$$
\begin{aligned}
r\left[\begin{array}{lll}
A & B & 0 \\
C & 0 & P
\end{array}\right] & =r(P)+r\left[\begin{array}{cc}
A & B \\
E_{P} C & 0
\end{array}\right], \\
r\left[\begin{array}{ll}
A & B \\
C & 0 \\
0 & Q
\end{array}\right] & =r(Q)+r\left[\begin{array}{cc}
A & B F_{Q} \\
C & 0
\end{array}\right], \\
r\left[\begin{array}{ccc}
A & B & 0 \\
C & 0 & P \\
0 & Q & 0
\end{array}\right] & =r(P)+r(Q)+r\left[\begin{array}{cc}
A & B F_{Q} \\
E_{P} C & 0
\end{array}\right] .
\end{aligned}
$$

We shall use them in Sections 3 and 4 to simplify ranks of block matrices involving $E_{P}$ and $F_{Q}$.

Lemma 2.3 ([27]). Let $A \in \mathbb{C}_{\mathrm{H}}^{m}, B \in \mathbb{C}^{m \times n}, D \in \mathbb{C}_{\mathrm{H}}^{n}$, and let

$$
U=\left[\begin{array}{cc}
A & B \\
B^{*} & 0
\end{array}\right], \quad V=\left[\begin{array}{cc}
A & B \\
B^{*} & D
\end{array}\right] .
$$

Then, the following expansion formulas hold

$$
\begin{aligned}
i_{ \pm}(U) & =r(B)+i_{ \pm}\left(E_{B} A E_{B}\right), \\
r(U) & =2 r(B)+r\left(E_{B} A E_{B}\right), \\
i_{ \pm}(V) & =i_{ \pm}(A)+i_{ \pm}\left[\begin{array}{cc}
0 & E_{A} B \\
B^{*} E_{A} & D-B^{*} A^{\dagger} B
\end{array}\right], \\
r(V) & =r(A)+r\left[\begin{array}{cc}
0 & E_{A} B \\
B^{*} E_{A} & D-B^{*} A^{\dagger} B
\end{array}\right] .
\end{aligned}
$$

In particular, the following hold.

(a) If $A \geqslant 0$, then

$$
i_{+}(U)=r[A, B], \quad i_{-}(U)=r(B), \quad r(U)=r[A, B]+r(B) .
$$

(b) If $A \leqslant 0$, then

$$
i_{+}(U)=r(B), \quad i_{-}(U)=r[A, B], \quad r(U)=r[A, B]+r(B) .
$$

(c) If $\mathscr{R}(B) \subseteq \mathscr{R}(A)$, then

$i_{ \pm}(V)=i_{ \pm}(A)+i_{ \pm}\left(D-B^{*} A^{\dagger} B\right), \quad r(V)=r(A)+r\left(D-B^{*} A^{\dagger} B\right)$.

(d) If $\mathscr{R}(B) \cap \mathscr{R}(A)=\{0\}$ and $\mathscr{R}\left(B^{*}\right) \cap \mathscr{R}(D)=\{0\}$, then

$$
i_{ \pm}(V)=i_{ \pm}(A)+i_{ \pm}(D)+r(B), \quad r(V)=r(A)+2 r(B)+r(D) .
$$

Three general expansion formulas derived from (2.7) and (2.8) are

$$
\begin{aligned}
& i_{ \pm}\left[\begin{array}{cc}
A & B F_{P} \\
F_{P} B^{*} & 0
\end{array}\right]=i_{ \pm}\left[\begin{array}{ccc}
A & B & 0 \\
B^{*} & 0 & P^{*} \\
0 & P & 0
\end{array}\right]-r(P), \\
& r\left[\begin{array}{cc}
A & B F_{P} \\
F_{P} B^{*} & 0
\end{array}\right]=r\left[\begin{array}{ccc}
A & B & 0 \\
B^{*} & 0 & P^{*} \\
0 & P & 0
\end{array}\right]-2 r(P) .
\end{aligned}
$$


We shall use them to simplify the inertias of block Hermitian matrices that involve $F_{P}=I-P^{\dagger} P$.

Lemma 2.4. Let $A_{j} \in \mathbb{C}^{m_{j} \times n}, B_{j} \in \mathbb{C}^{p \times q_{j}}$ and $C_{j} \in \mathbb{C}^{m_{j} \times q_{j}}$ be given, $j=1,2$. Then, the following hold.

(a) [24] The pair of matrix equations

$$
A_{1} X B_{1}=C_{1} \text { and } A_{2} X B_{2}=C_{2}
$$

have a common solution for $X \in \mathbb{C}^{n \times p}$ if and only if

$$
\begin{aligned}
& \mathscr{R}\left(C_{j}\right) \subseteq \mathscr{R}\left(A_{j}\right), \quad \mathscr{R}\left(C_{j}^{*}\right) \subseteq \mathscr{R}\left(B_{j}^{*}\right), \quad j=1,2, \\
& r\left[\begin{array}{ccc}
C_{1} & 0 & A_{1} \\
0 & -C_{2} & A_{2} \\
B_{1} & B_{2} & 0
\end{array}\right]=r\left[\begin{array}{l}
A_{1} \\
A_{2}
\end{array}\right]+r\left[B_{1}, B_{2}\right] .
\end{aligned}
$$

(b) [26] Under (2.18) and (2.19), the general common solution of (2.17) can be written in the following parametric form

$$
X=X_{0}+F_{A} V_{1}+V_{2} E_{B}+F_{A_{1}} V_{3} E_{B_{2}}+F_{A_{2}} V_{4} E_{B_{1}},
$$

where $A=\left[\begin{array}{l}A_{1} \\ A_{2}\end{array}\right], B=\left[B_{1}, B_{2}\right]$, and the four matrices $V_{1}, \ldots, V_{4} \in \mathbb{C}^{n \times p}$ are arbitrary.

Lemma 2.5. Let $A \in \mathbb{C}^{m \times n}$ and $B \in \mathbb{C}_{\mathrm{H}}^{m}$ be given. Then, the following hold.

(a) $[5,9]$ The matrix equation $A X A^{*}=B$ has a solution $X \in \mathbb{C}_{\mathrm{H}}^{n}$ if and only if $\mathscr{R}(B) \subseteq \mathscr{R}(A)$, or equivalently, $A A^{\dagger} B=B$.

(b) [27] Under $A A^{\dagger} B=B$, the general Hermitian solution of $A X A^{*}=B$ can be written in the following two forms

$$
\begin{aligned}
& X=A^{\dagger} B\left(A^{\dagger}\right)^{*}+U-A^{\dagger} A U A^{\dagger} A, \\
& X=A^{\dagger} B\left(A^{\dagger}\right)^{*}+F_{A} V+V^{*} F_{A},
\end{aligned}
$$

where $U \in \mathbb{C}_{\mathrm{H}}^{n}$ and $V \in \mathbb{C}^{n \times n}$ are arbitrary.

More results on properties of solutions of $A X A^{*}=B$ can be found in $[14,18]$.

Lemma 2.6 ([9]). Let $A, B \in \mathbb{C}^{m \times n}$ be given. Then, the following hold.

(a) The matrix equation $A X=B$ has a Hermitian solution $X \in \mathbb{C}_{\mathrm{H}}^{n}$ if and only if $\mathscr{R}(B) \subseteq \mathscr{R}(A)$ and $A B^{*}=B A^{*}$. In this case, the general Hermitian solution of $A X=B$ can be written as

$$
X=A^{\dagger} B+\left(A^{\dagger} B\right)^{*}-A^{\dagger} B A^{\dagger} A+F_{A} U F_{A},
$$

where $U \in \mathbb{C}_{\mathrm{H}}^{n}$ is arbitrary.

(b) The matrix equation $A X=B$ has a solution $0 \leqslant X \in \mathbb{C}_{\mathrm{H}}^{n}$ if and only if $\mathscr{R}(B) \subseteq \mathscr{R}(A), A B^{*} \geqslant 0$ and $r\left(A B^{*}\right)=r(B)$. In this case, the general positive semi-definite solution of $A X=B$ can be written as

$$
X=B^{*}\left(A B^{*}\right)^{\dagger} B+F_{A} U F_{A},
$$

where $0 \leqslant U \in \mathbb{C}_{\mathrm{H}}^{n}$ is arbitrary. 
Lemma 2.7. Let $A \in \mathbb{C}_{\mathrm{H}}^{m}$ and $B \in \mathbb{C}^{m \times n}$ be given, and let $M=\left[\begin{array}{cc}A & B \\ B^{*} & 0\end{array}\right]$. Then, the following hold.

(a) [27, 39] The global maximal and minimal ranks and inertias of the Hermitian matrix-valued function $A-B X B^{*}$ subject to $X \in \mathbb{C}_{\mathrm{H}}^{n}$ are given by

$$
\begin{aligned}
\max _{X \in \mathbb{C}_{\mathrm{H}}^{n}} r\left(A-B X B^{*}\right) & =r[A, B], \\
\min _{X \in \mathbb{C}_{\mathrm{H}}^{n}} r\left(A-B X B^{*}\right) & =2 r[A, B]-r(M), \\
\max _{X \in \mathbb{C}_{\mathrm{H}}^{n}} i_{ \pm}\left(A-B X B^{*}\right) & =i_{ \pm}(M), \\
\min _{X \in \mathbb{C}_{\mathrm{H}}^{n}} i_{ \pm}\left(A-B X B^{*}\right) & =r[A, B]-i_{\mp}(M) .
\end{aligned}
$$

(b) [32] The global maximal and minimal ranks and inertias of the Hermitian matrix-valued functions $A \pm B X B^{*}$ subject to $0 \leqslant X \in \mathbb{C}_{\mathrm{H}}^{n}$ are given by

$$
\begin{aligned}
\max _{0 \leqslant X \in \mathbb{C}_{\mathrm{H}}^{n}} r\left(A+B X B^{*}\right) & =r[A, B], \\
\min _{0 \leqslant X \in \mathbb{C}_{\mathrm{H}}^{n}} r\left(A+B X B^{*}\right) & =i_{+}(A)+r[A, B]-i_{+}(M), \\
\max _{0 \leqslant X \in \mathbb{C}_{\mathrm{H}}^{n}} i_{+}\left(A+B X B^{*}\right) & =i_{+}(M), \\
\min _{0 \leqslant X \in \mathbb{C}_{\mathrm{H}}^{n}} i_{+}\left(A+B X B^{*}\right) & =i_{+}(A), \\
\max _{0 \leqslant X \in \mathbb{C}_{\mathrm{H}}^{n}} i_{-}\left(A+B X B^{*}\right) & =i_{-}(A), \\
\min _{0 \leqslant X \in \mathbb{C}_{\mathrm{H}}^{n}} i_{-}\left(A+B X B^{*}\right) & =r[A, B]-i_{+}(M), \\
\max _{0 \leqslant X \in \mathbb{C}_{\mathrm{H}}^{n}} r\left(A-B X B^{*}\right) & =r[A, B], \\
\min _{0 \leqslant X \in \mathbb{C}_{\mathrm{H}}^{n}} r\left(A-B X B^{*}\right) & =i_{-}(A)+r[A, B]-i_{-}(M), \\
\max _{0 \leqslant X \in \mathbb{C}_{\mathrm{H}}^{n}} i_{+}\left(A-B X B^{*}\right) & =i_{+}(A), \\
\min _{0 \leqslant X \in \mathbb{C}_{\mathrm{H}}^{n}} i_{+}\left(A-B X B^{*}\right) & =r[A, B]-i_{-}(M), \\
\max _{0 \leqslant X \in \mathbb{C}_{\mathrm{H}}^{n} i_{-}}\left(A-B X B^{*}\right) & =i_{-}(M), \\
\min _{0 \leqslant X \in \mathbb{C}_{\mathrm{H}}^{n} i_{-}}\left(A-B X B^{*}\right) & =i_{-}(A) .
\end{aligned}
$$

Lemma 2.8 ([16]). Let $A \in \mathbb{C}_{\mathrm{H}}^{m}, B \in \mathbb{C}^{m \times p}$ and $C \in \mathbb{C}^{q \times m}$ be given, and let

$$
\begin{array}{ll}
M_{1}=\left[\begin{array}{cc}
A & B \\
B^{*} & 0
\end{array}\right], & M_{2}=\left[\begin{array}{cc}
A & C^{*} \\
C & 0
\end{array}\right], \\
N=\left[A, B, C^{*}\right], & N_{1}=\left[\begin{array}{ccc}
A & B & C^{*} \\
B^{*} & 0 & 0
\end{array}\right], \quad N_{2}=\left[\begin{array}{ccc}
A & B & C^{*} \\
C & 0 & 0
\end{array}\right] .
\end{array}
$$


Then, the global maximal and minimal ranks and inertias of the Hermitian matrixvalued function $A-B X C-(B X C)^{*}$ are given by

$$
\begin{aligned}
& \max _{X \in \mathbb{C}^{p \times q}} r\left[A-B X C-(B X C)^{*}\right]=\min \left\{r(N), \quad r\left(M_{1}\right), \quad r\left(M_{2}\right)\right\}, \\
& \min _{X \in \mathbb{C}^{p \times q}} r\left[A-B X C-(B X C)^{*}\right]=2 r(N)+\max \left\{s_{1}, \quad s_{2}, s_{3}, \quad s_{4}\right\}, \\
& \max _{X \in \mathbb{C}^{p \times q}} i_{ \pm}\left[A-B X C-(B X C)^{*}\right]=\min \left\{i_{ \pm}\left(M_{1}\right), i_{ \pm}\left(M_{2}\right)\right\}, \\
& \min _{X \in \mathbb{C}^{p \times q}} i_{ \pm}\left[A-B X C-(B X C)^{*}\right]=r(N)+\max \left\{i_{ \pm}\left(M_{1}\right)-r\left(N_{1}\right),\right. \\
& \left.i_{ \pm}\left(M_{2}\right)-r\left(N_{2}\right)\right\}
\end{aligned}
$$

where

$$
\begin{gathered}
s_{1}=r\left(M_{1}\right)-2 r\left(N_{1}\right), \quad s_{2}=r\left(M_{2}\right)-2 r\left(N_{2}\right), \\
s_{3}=i_{+}\left(M_{1}\right)+i_{-}\left(M_{2}\right)-r\left(N_{1}\right)-r\left(N_{2}\right), \quad s_{4}=i_{-}\left(M_{1}\right)+i_{+}\left(M_{2}\right)-r\left(N_{1}\right)-r\left(N_{2}\right) .
\end{gathered}
$$

In particular, if $\mathscr{R}\left(C^{*}\right) \subseteq \mathscr{R}(B)$, then

$$
\begin{aligned}
\max _{X \in \mathbb{C}^{p \times q}} r\left[A-B X C-(B X C)^{*}\right] & =\min \left\{r[A, B], \quad r\left[\begin{array}{cc}
A & C^{*} \\
C & 0
\end{array}\right]\right\} \\
\min _{X \in \mathbb{C}^{p \times q}} r\left[A-B X C-(B X C)^{*}\right] & =2 r[A, B]+r\left[\begin{array}{cc}
A & C^{*} \\
C & 0
\end{array}\right]-2 r\left[\begin{array}{cc}
A & B \\
C & 0
\end{array}\right], \\
\max _{X \in \mathbb{C}^{p \times q}} i_{ \pm}\left[A-B X C-(B X C)^{*}\right] & =i_{ \pm}\left[\begin{array}{cc}
A & C^{*} \\
C & 0
\end{array}\right], \\
\min _{X \in \mathbb{C}^{p \times q}} i_{ \pm}\left[A-B X C-(B X C)^{*}\right] & =r[A, B]+i_{ \pm}\left[\begin{array}{cc}
A & C^{*} \\
C & 0
\end{array}\right]-r\left[\begin{array}{cc}
A & B \\
C & 0
\end{array}\right] .
\end{aligned}
$$

The matrices $X$ that satisfy (2.43)-(2.50) (namely, the global maximizers and minimizers of the objective rank and inertia functions) are not necessarily unique and their expressions were also given in [16] by using certain simultaneous decomposition of the three given matrices and discrete methods. Observe that the right-hand sides of (2.43)-(2.50) are represented in analytical forms of the ranks and inertias of the five given block matrices, we can easily use them to derive extremal ranks and inertias of some general linear and nonlinear matrix-valued functions. In these cases, combining the rank and inertia formulas obtained with the assertions in Lemma 2.1 may yield various conclusions on algebraic properties of linear and nonlinear matrix-valued functions.

\section{The extremal Ranks and inertias of $A-B_{1} X B_{1}^{*}$ Subject to}

$$
B_{2} X B_{2}^{*}=A_{2} \text { AND } B_{3} X B_{3}^{*}=A_{3}
$$

We first derive a parametric form for the general common Hermitian solution of the pair of matrix equations in (1.2).

Lemma 3.1 ([31]). Let $A_{i} \in \mathbb{C}_{\mathrm{H}}^{m_{i}}, B_{i} \in \mathbb{C}^{m_{i} \times n}$ be given for $i=2,3$, and suppose that each of the two matrix equations

$$
B_{2} X B_{2}^{*}=A_{2} \text { and } B_{3} X B_{3}^{*}=A_{3}
$$

has a solution, i.e., $\mathscr{R}\left(A_{i}\right) \subseteq \mathscr{R}\left(B_{i}\right)$ for $i=2$, 3. Then, the following hold. 
(a) The pair of matrix equations have a common Hermitian solution if and only if

$$
r\left[\begin{array}{ccc}
A_{2} & 0 & B_{2} \\
0 & -A_{3} & B_{3} \\
B_{2}^{*} & B_{3}^{*} & 0
\end{array}\right]=2 r\left[\begin{array}{l}
B_{2} \\
B_{3}
\end{array}\right] .
$$

(b) Under (3.2), the general common Hermitian solution of the pair of equations can be written in the following parametric form

$$
X=X_{0}+V F_{B}+F_{B} V^{*}+F_{B_{2}} U F_{B_{3}}+F_{B_{3}} U^{*} F_{B_{2}}
$$

where $X_{0}$ is a special Hermitian common solution to the pair of equations, $B=\left[\begin{array}{l}B_{2} \\ B_{3}\end{array}\right]$, and $U, V \in \mathbb{C}^{n \times n}$ are arbitrary.

Substituting (3.3) into $A_{1}-B_{1} X B_{1}^{*}$ gives

$$
\begin{aligned}
A_{1}-B_{1} X B_{1}^{*}= & A_{1}-B_{1} X_{0} B_{1}^{*}-B_{1} V F_{B} B_{1}^{*} \\
& -B_{1} F_{B} V^{*} B_{1}^{*}-B_{1} F_{B_{2}} U F_{B_{3}} B_{1}^{*}-B_{1} F_{B_{3}} U^{*} F_{B_{2}} B_{1}^{*},
\end{aligned}
$$

which is a matrix-valued function involving two variable matrices $V$ and $U$. Thus, the matrix-valued function $A_{1}-B_{1} X B_{1}^{*}$ subject to (1.2) is equivalently converted to the unconstrained matrix-valued function in (3.4). To find the global maximal and minimal ranks and inertias of (3.4), we need the following result.

Lemma 3.2. Let

$$
\phi\left(X_{1}, X_{2}\right)=A-B_{1} X_{1} C_{1}-\left(B_{1} X_{1} C_{1}\right)^{*}-B_{2} X_{2} C_{2}-\left(B_{2} X_{2} C_{2}\right)^{*},
$$

where $A \in \mathbb{C}_{\mathrm{H}}^{m}, B_{i} \in \mathbb{C}^{m \times p_{i}}$ and $C_{i} \in \mathbb{C}^{q_{i} \times m}$ are given, and $X_{i} \in \mathbb{C}^{p_{i} \times q_{i}}$ are variable matrices for $i=1,2$, and assume that

$$
\mathscr{R}\left(B_{2}\right) \subseteq \mathscr{R}\left(B_{1}\right), \quad \mathscr{R}\left(C_{1}^{*}\right) \subseteq \mathscr{R}\left(B_{1}\right), \quad \mathscr{R}\left(C_{2}^{*}\right) \subseteq \mathscr{R}\left(B_{1}\right)
$$

Also let

$$
\begin{aligned}
& N=\left[\begin{array}{cccc}
A & B_{2} & C_{1}^{*} & C_{2}^{*} \\
C_{1} & 0 & 0 & 0
\end{array}\right], N_{1}=\left[\begin{array}{cccc}
A & B_{2} & C_{1}^{*} & C_{2}^{*} \\
B_{2}^{*} & 0 & 0 & 0 \\
C_{1} & 0 & 0 & 0
\end{array}\right], N_{2}=\left[\begin{array}{cccc}
A & B_{2} & C_{1}^{*} & C_{2}^{*} \\
C_{1} & 0 & 0 & 0 \\
C_{2} & 0 & 0 & 0
\end{array}\right], \\
& M=\left[\begin{array}{cc}
A & B_{1} \\
C_{1} & 0
\end{array}\right], M_{1}=\left[\begin{array}{ccc}
A & B_{2} & C_{1}^{*} \\
B_{2}^{*} & 0 & 0 \\
C_{1} & 0 & 0
\end{array}\right], M_{2}=\left[\begin{array}{ccc}
A & C_{1}^{*} & C_{2}^{*} \\
C_{1} & 0 & 0 \\
C_{2} & 0 & 0
\end{array}\right] .
\end{aligned}
$$


Then, the global maximal and minimal ranks and inertias of $\phi\left(X_{1}, X_{2}\right)$ are given by

$$
\begin{aligned}
& \max _{X_{1} \in \mathbb{C}^{p_{1} \times q_{1}}, X_{2} \in \mathbb{C}^{p_{2} \times q_{2}}} r\left[\phi\left(X_{1}, X_{2}\right)\right]=\min \left\{r\left[A, B_{1}\right], r(N), r\left(M_{1}\right), r\left(M_{2}\right)\right\}, \\
& \min _{X_{1} \in \mathbb{C}^{p_{1} \times q_{1}}, X_{2} \in \mathbb{C}^{p_{2} \times q_{2}}} r\left[\phi\left(X_{1}, X_{2}\right)\right]=2 r\left[A, B_{1}\right]-2 r(M)+2 r(N) \\
& +\max \left\{s_{1}, s_{2}, s_{3}, s_{4}\right\}, \\
& \max _{X_{1} \in \mathbb{C}^{p_{1} \times q_{1}}, X_{2} \in \mathbb{C}^{p_{2} \times q_{2}}} i_{ \pm}\left[\phi\left(X_{1}, X_{2}\right)\right]=\min \left\{i_{ \pm}\left(M_{1}\right), \quad i_{ \pm}\left(M_{2}\right)\right\} \\
& \min _{X_{1} \in \mathbb{C}^{p_{1} \times q_{1}}, X_{2} \in \mathbb{C}^{p_{2} \times q_{2}}} i_{ \pm}\left[\phi\left(X_{1}, X_{2}\right)\right]=r\left[A, B_{1}\right]-r(M)+r(N) \\
& +\max \left\{i_{ \pm}\left(M_{1}\right)-r\left(N_{1}\right), \quad i_{ \pm}\left(M_{2}\right)-r\left(N_{2}\right)\right\}
\end{aligned}
$$

where

$$
\begin{aligned}
& s_{1}=r\left(M_{1}\right)-2 r\left(N_{1}\right), \quad s_{2}=r\left(M_{2}\right)-2 r\left(N_{2}\right), \\
& s_{3}=i_{+}\left(M_{1}\right)+i_{-}\left(M_{2}\right)-r\left(N_{1}\right)-r\left(N_{2}\right), \\
& s_{4}=i_{-}\left(M_{1}\right)+i_{+}\left(M_{2}\right)-r\left(N_{1}\right)-r\left(N_{2}\right) .
\end{aligned}
$$

Proof. Under (3.6), applying (2.47)-(2.50) to the variable matrix $X_{1}$ in (3.5) and simplifying, we obtain

$$
\begin{aligned}
& \max _{X_{1} \in \mathbb{C}^{p_{1} \times q_{1}}} r\left[\phi\left(X_{1}, X_{2}\right)\right] \\
& =\min \left\{r\left[A-B_{2} X_{2} C_{2}-\left(B_{2} X_{2} C_{2}\right)^{*}, B_{1}\right], r\left[\begin{array}{cc}
A-B_{2} X_{2} C_{2}-\left(B_{2} X_{2} C_{2}\right)^{*} & C_{1}^{*} \\
C_{1} & 0
\end{array}\right]\right\} \\
& =\min \left\{r\left[A, B_{1}\right], \quad r\left[\begin{array}{cc}
A-B_{2} X_{2} C_{2}-\left(B_{2} X_{2} C_{2}\right)^{*} & C_{1}^{*} \\
C_{1} & 0
\end{array}\right]\right\} \text {, } \\
& \min _{X_{1} \in \mathbb{C}^{p_{1} \times q_{1}}} r\left[\phi\left(X_{1}, X_{2}\right)\right] \\
& =2 r\left[A-B_{2} X_{2} C_{2}-\left(B_{2} X_{2} C_{2}\right)^{*}, B_{1}\right]+r\left[\begin{array}{cc}
A-B_{2} X_{2} C_{2}-\left(B_{2} X_{2} C_{2}\right)^{*} & C_{1}^{*} \\
C_{1} & 0
\end{array}\right] \\
& -2 r\left[\begin{array}{cc}
A-B_{2} X_{2} C_{2}-\left(B_{2} X_{2} C_{2}\right)^{*} & B_{1} \\
C_{1} & 0
\end{array}\right] \\
& =2 r\left[A, B_{1}\right]+r\left[\begin{array}{cc}
A-B_{2} X_{2} C_{2}-\left(B_{2} X_{2} C_{2}\right)^{*} & C_{1}^{*} \\
C_{1} & 0
\end{array}\right]-2 r\left[\begin{array}{cc}
A & B_{1} \\
C_{1} & 0
\end{array}\right],
\end{aligned}
$$




$$
\begin{aligned}
& \max _{X_{1} \in \mathbb{C}^{p_{1} \times q_{1}}} i_{ \pm}\left[\phi\left(X_{1}, X_{2}\right)\right]=i_{ \pm}\left[\begin{array}{rr}
A-B_{2} X_{2} C_{2}-\left(B_{2} X_{2} C_{2}\right)^{*} & C_{1}^{*} \\
C_{1} & 0
\end{array}\right], \\
& \min _{X_{1} \in \mathbb{C}^{p_{1} \times q_{1}}} i_{ \pm}\left[\phi\left(X_{1}, X_{2}\right)\right] \\
& =r\left[A-B_{2} X_{2} C_{2}-\left(B_{2} X_{2} C_{2}\right)^{*}, B_{1}\right]+i_{ \pm}\left[\begin{array}{rr}
A-B_{2} X_{2} C_{2}-\left(B_{2} X_{2} C_{2}\right)^{*} & C_{1}^{*} \\
& C_{1}
\end{array}\right] \\
& -r\left[\begin{array}{rr}
A-B_{2} X_{2} C_{2}-\left(B_{2} X_{2} C_{2}\right)^{*} & B_{1} \\
C_{1} & 0
\end{array}\right] \\
& =r\left[A, B_{1}\right]+i_{ \pm}\left[\begin{array}{rr}
A-B_{2} X_{2} C_{2}-\left(B_{2} X_{2} C_{2}\right)^{*} & C_{1}^{*} \\
C_{1} & 0
\end{array}\right]-r\left[\begin{array}{cc}
A & B_{1} \\
C_{1} & 0
\end{array}\right] .
\end{aligned}
$$

Notice that

$$
\begin{aligned}
& {\left[\begin{array}{cc}
A-B_{2} X_{2} C_{2}-\left(B_{2} X_{2} C_{2}\right)^{*} & C_{1}^{*} \\
C_{1} & 0
\end{array}\right]} \\
& =\left[\begin{array}{cc}
A & C_{1}^{*} \\
C_{1} & 0
\end{array}\right]-\left[\begin{array}{c}
B_{2} \\
0
\end{array}\right] X_{2}\left[C_{2}, 0\right]-\left[\begin{array}{c}
C_{2}^{*} \\
0
\end{array}\right] X_{2}^{*}\left[B_{2}^{*}, 0\right]:=\psi\left(X_{2}\right) .
\end{aligned}
$$

Applying (2.43)-(2.46) to this matrix-valued function $\psi\left(X_{2}\right)$ gives

$$
\begin{aligned}
\max _{X_{2} \in \mathbb{C}^{p_{2}} \times q_{2}} r\left[\psi\left(X_{2}\right)\right] & =\min \left\{r(N), \quad r\left(M_{1}\right), \quad r\left(M_{2}\right)\right\}, \\
\min _{X_{2} \in \mathbb{C}^{p_{2} \times q_{2}}} r\left[\psi\left(X_{2}\right)\right] & =2 r(N)+\max \left\{s_{1}, s_{2}, s_{3}, s_{4}\right\}, \\
\max _{X_{2} \in \mathbb{C}^{p_{2} \times q_{2}}} i_{ \pm}\left[\psi\left(X_{2}\right)\right] & =\min \left\{i_{ \pm}\left(M_{1}\right), i_{ \pm}\left(M_{2}\right)\right\}, \\
\min _{X_{2} \in \mathbb{C}^{p_{2} \times q_{2}}} i_{ \pm}\left[\psi\left(X_{2}\right)\right] & =r(N)+\max \left\{i_{ \pm}\left(M_{1}\right)-r\left(N_{1}\right), i_{ \pm}\left(M_{2}\right)-r\left(N_{2}\right)\right\},
\end{aligned}
$$

where

$$
\begin{gathered}
s_{1}=r\left(M_{1}\right)-2 r\left(N_{1}\right), \quad s_{2}=r\left(M_{2}\right)-2 r\left(N_{2}\right), \\
s_{3}=i_{+}\left(M_{1}\right)+i_{-}\left(M_{2}\right)-r\left(N_{1}\right)-r\left(N_{2}\right), \quad s_{4}=i_{-}\left(M_{1}\right)+i_{+}\left(M_{2}\right)-r\left(N_{1}\right)-r\left(N_{2}\right) .
\end{gathered}
$$

Substituting these results into (3.11)-(3.14) yields (3.7)-(3.10).

For convenience of representation, we rewrite (3.4) as

$$
A_{1}-B_{1} X B_{1}^{*}=A-G_{1} V G_{2}-\left(G_{1} V G_{2}\right)^{*}-G_{3} U G_{4}-\left(G_{3} U G_{4}\right)^{*}
$$

where

$$
A=A_{1}-B_{1} X_{0} B_{1}^{*}, G_{1}=B_{1}, G_{2}=F_{B} B_{1}^{*}, G_{3}=B_{1} F_{B_{2}}, G_{4}=F_{B_{3}} B_{1}^{*} .
$$

It is easy to verify that the above matrices satisfy the conditions

$$
\begin{gathered}
\mathscr{R}\left(G_{2}^{*}\right) \subseteq \mathscr{R}\left(G_{1}\right), \quad \mathscr{R}\left(G_{3}\right) \subseteq \mathscr{R}\left(G_{1}\right), \quad \mathscr{R}\left(G_{4}^{*}\right) \subseteq \mathscr{R}\left(G_{1}\right), \\
\mathscr{R}\left(G_{2}^{*}\right) \subseteq \mathscr{R}\left(G_{3}\right), \quad \mathscr{R}\left(G_{2}^{*}\right) \subseteq \mathscr{R}\left(G_{4}^{*}\right) .
\end{gathered}
$$

So that (3.20) is special case of (3.5) under (3.6). In this case, applying Lemma 3.2 to (3.20) yields the main results of this section. 
Theorem 3.3. Let $A_{i} \in \mathbb{C}_{\mathrm{H}}^{m_{i}}$ and $B_{i} \in \mathbb{C}^{m_{i} \times n}$ be given for $i=1,2,3$, and assume that the pair of matrix equations

$$
B_{2} X B_{2}^{*}=A_{2} \text { and } B_{3} X B_{3}^{*}=A_{3}
$$

have a common solution $X \in \mathbb{C}_{\mathrm{H}}^{n}$. Also let $\mathcal{S}$ be defined in (1.2) and define

$$
\begin{array}{rlrl}
P_{1}= & {\left[\begin{array}{cccc}
A_{1} & B_{1} & 0 & 0 \\
B_{1}^{*} & 0 & B_{2}^{*} & B_{3}^{*}
\end{array}\right],} & P_{2} & =\left[\begin{array}{ccc}
A_{1} & 0 & B_{1} \\
0 & -A_{2} & B_{2} \\
B_{1}^{*} & B_{2}^{*} & 0
\end{array}\right], \\
P_{3}=\left[\begin{array}{ccc}
A_{1} & 0 & B_{1} \\
0 & -A_{3} & B_{3} \\
B_{1}^{*} & B_{3}^{*} & 0
\end{array}\right], & Q_{1}=\left[\begin{array}{ccccc}
A_{1} & 0 & 0 & B_{1} & B_{1} \\
0 & -A_{2} & 0 & B_{2} & 0 \\
0 & 0 & -A_{3} & 0 & B_{3} \\
B_{1}^{*} & B_{2}^{*} & B_{3}^{*} & 0 & 0
\end{array}\right], \\
Q_{2}=\left[\begin{array}{cccc}
A_{1} & 0 & B_{1} & B_{1} \\
0 & -A_{2} & B_{2} & 0 \\
B_{1}^{*} & B_{2}^{*} & 0 & 0 \\
0 & 0 & 0 & B_{3}
\end{array}\right], & Q_{3}=\left[\begin{array}{cccc}
A_{1} & 0 & B_{1} & B_{1} \\
0 & -A_{3} & B_{3} & 0 \\
B_{1}^{*} & B_{3}^{*} & 0 & 0 \\
0 & 0 & 0 & B_{2}
\end{array}\right]
\end{array}
$$

Then, the following hold.

(a) The global maximal rank of $A_{1}-B_{1} X B_{1}^{*}$ subject to $\mathcal{S}$ in (1.2) is

$$
\begin{aligned}
\max _{X \in \mathcal{S}} r\left(A_{1}-B_{1} X B_{1}^{*}\right)=\min & \left\{r\left[A_{1}, B_{1}\right], r\left(Q_{1}\right)-r\left[\begin{array}{c}
B_{2} \\
B_{3}
\end{array}\right]-r\left(B_{2}\right)-r\left(B_{3}\right)\right. \\
& \left.r\left(P_{2}\right)-2 r\left(B_{2}\right), r\left(P_{3}\right)-2 r\left(B_{3}\right)\right\} .
\end{aligned}
$$

(b) The global minimal rank of $A_{1}-B_{1} X B_{1}^{*}$ subject to $\mathcal{S}$ in (1.2) is

$$
\begin{aligned}
\min _{X \in \mathcal{S}} r\left(A_{1}-B_{1} X B_{1}^{*}\right) & =2 r\left[A_{1}, B_{1}\right]-2 r\left(P_{1}\right)+2 r\left(Q_{1}\right) \\
& +\max \left\{r\left(P_{2}\right)-2 r\left(Q_{2}\right), r\left(P_{3}\right)-2 r\left(Q_{3}\right), u_{1}, u_{2}\right\},
\end{aligned}
$$

where

$$
\begin{aligned}
& u_{1}=i_{+}\left(P_{2}\right)+i_{-}\left(P_{3}\right)-r\left(Q_{2}\right)-r\left(Q_{3}\right), \\
& u_{2}=i_{-}\left(P_{2}\right)+i_{+}\left(P_{3}\right)-r\left(Q_{2}\right)-r\left(Q_{3}\right) .
\end{aligned}
$$

(c) The global maximal inertia of $A_{1}-B_{1} X B_{1}^{*}$ subject to $\mathcal{S}$ in (1.2) is

$$
\max _{X \in \mathcal{S}} i_{ \pm}\left(A_{1}-B_{1} X B_{1}^{*}\right)=\min \left\{i_{ \pm}\left(P_{2}\right)-r\left(B_{2}\right), \quad i_{ \pm}\left(P_{3}\right)-r\left(B_{3}\right)\right\} .
$$

(d) The global minimal inertia of $A_{1}-B_{1} X B_{1}^{*}$ subject to $\mathcal{S}$ in (1.2) is

$$
\begin{aligned}
\min _{X \in \mathcal{S}} i_{ \pm}\left(A_{1}-B_{1} X B_{1}^{*}\right)= & r\left[A_{1}, B_{1}\right]-r\left(P_{1}\right)+r\left(Q_{1}\right) \\
& +\max \left\{i_{ \pm}\left(P_{2}\right)-r\left(Q_{2}\right), i_{ \pm}\left(P_{3}\right)-r\left(Q_{3}\right)\right\} .
\end{aligned}
$$


Proof. Under (3.23), we find by Lemma 3.2 that

$$
\begin{aligned}
& \max _{X \in \mathcal{S}} r\left(A_{1}-B_{1} X B_{1}^{*}\right) \\
& =\max _{V, U} r\left[A-G_{1} V G_{2}-\left(G_{1} V G_{2}\right)^{*}-G_{3} U G_{4}-\left(G_{3} U G_{4}\right)^{*}\right] \\
& =\min \left\{r\left[A, G_{1}\right], r\left[\begin{array}{ccc}
A & G_{3} & G_{4}^{*} \\
G_{2} & 0 & 0
\end{array}\right], r\left[\begin{array}{cc}
A & G_{3} \\
G_{3}^{*} & 0
\end{array}\right], r\left[\begin{array}{cc}
A & G_{4}^{*} \\
G_{4} & 0
\end{array}\right]\right\}, \\
& \min _{X \in \mathcal{S}} r\left(A_{1}-B_{1} X B_{1}^{*}\right) \\
& =\min _{V, U} r\left[A-G_{1} V G_{2}-\left(G_{1} V G_{2}\right)^{*}-G_{3} U G_{4}-\left(G_{3} U G_{4}\right)^{*}\right] \\
& =2 r\left[A, G_{1}\right]-2 r\left[\begin{array}{cc}
A & G_{1} \\
G_{2} & 0
\end{array}\right]+2 r\left[\begin{array}{ccc}
A & G_{3} & G_{4}^{*} \\
G_{2} & 0 & 0
\end{array}\right] \\
& +\max \left\{s_{1}, s_{2}, s_{3}, s_{4}\right\}, \\
& \max _{X \in \mathcal{S}} i_{ \pm}\left(A_{1}-B_{1} X B_{1}^{*}\right) \\
& =\max _{V, U} i_{ \pm}\left[A-G_{1} V G_{2}-\left(G_{1} V G_{2}\right)^{*}-G_{3} U G_{4}-\left(G_{3} U G_{4}\right)^{*}\right] \\
& =\min \left\{i_{ \pm}\left[\begin{array}{cc}
A & G_{3} \\
G_{3}^{*} & 0
\end{array}\right], i_{ \pm}\left[\begin{array}{cc}
A & G_{4}^{*} \\
G_{4} & 0
\end{array}\right]\right\} \\
& \min _{X \in \mathcal{S}} i_{ \pm}\left(A_{1}-B_{1} X B_{1}^{*}\right) \\
& =\min _{V, U} i_{ \pm}\left[A-G_{1} V G_{2}-\left(G_{1} V G_{2}\right)^{*}-G_{3} U G_{4}-\left(G_{3} U G_{4}\right)^{*}\right] \\
& =r\left[A, G_{1}\right]-r\left[\begin{array}{cc}
A & G_{1} \\
G_{2} & 0
\end{array}\right]+r\left[\begin{array}{ccc}
A & G_{3} & G_{4}^{*} \\
G_{2} & 0 & 0
\end{array}\right]+\max \left\{t_{1}, t_{2}\right\},
\end{aligned}
$$

where

$$
\begin{aligned}
s_{1} & =r\left[\begin{array}{cc}
A & G_{3} \\
G_{3}^{*} & 0
\end{array}\right]-2 r\left[\begin{array}{ccc}
A & G_{3} & G_{4}^{*} \\
G_{3}^{*} & 0 & 0
\end{array}\right], \\
s_{2} & =r\left[\begin{array}{cc}
A & G_{4}^{*} \\
G_{4} & 0
\end{array}\right]-2 r\left[\begin{array}{ccc}
A & G_{3} & G_{4}^{*} \\
G_{4} & 0 & 0
\end{array}\right], \\
s_{3} & =i_{+}\left[\begin{array}{cc}
A & G_{3} \\
G_{3}^{*} & 0
\end{array}\right]+i_{-}\left[\begin{array}{cc}
A & G_{4}^{*} \\
G_{4} & 0
\end{array}\right]-r\left[\begin{array}{ccc}
A & G_{3} & G_{4}^{*} \\
G_{3}^{*} & 0 & 0
\end{array}\right]-r\left[\begin{array}{ccc}
A & G_{3} & G_{4}^{*} \\
G_{4} & 0 & 0
\end{array}\right], \\
s_{4} & =i_{-}\left[\begin{array}{cc}
A & G_{3} \\
G_{3}^{*} & 0
\end{array}\right]+i_{+}\left[\begin{array}{cc}
A & G_{4}^{*} \\
G_{4} & 0
\end{array}\right]-r\left[\begin{array}{ccc}
A & G_{3} & G_{4}^{*} \\
G_{3}^{*} & 0 & 0
\end{array}\right]-r\left[\begin{array}{ccc}
A & G_{3} & G_{4}^{*} \\
G_{4} & 0 & 0
\end{array}\right], \\
t_{1} & =i_{ \pm}\left[\begin{array}{cc}
A & G_{3} \\
G_{3}^{*} & 0
\end{array}\right]-r\left[\begin{array}{ccc}
A & G_{3} & G_{4}^{*} \\
G_{3}^{*} & 0 & 0
\end{array}\right], \\
t_{2} & =i_{ \pm}\left[\begin{array}{cc}
A & G_{4} \\
G_{4}^{*} & 0
\end{array}\right]-r\left[\begin{array}{ccc}
A & G_{3} & G_{4}^{*} \\
G_{4}^{*} & 0 & 0
\end{array}\right] .
\end{aligned}
$$

Applying (2.4)-(2.6), (2.15) and (2.16), and simplifying by $\left[B_{2} X_{0} B_{2}^{*}, B_{3} X_{0} B_{3}^{*}\right]=$ $\left[A_{2}, A_{3}\right]$, elementary matrix operations and congruence matrix operations, we 
obtain

$$
\begin{aligned}
& r\left[A, G_{1}\right]=r\left[A_{1}-B_{1} X_{0} B_{1}^{*}, B_{1}\right]=r\left[A_{1}, B_{1}\right], \\
& r\left[\begin{array}{ccc}
A & G_{3} & G_{4}^{*} \\
G_{2} & 0 & 0
\end{array}\right]=r\left[\begin{array}{ccc}
A_{1}-B_{1} X_{0} B_{1}^{*} & B_{1} F_{B_{2}} & B_{1} F_{B_{3}} \\
F_{B} B_{1}^{*} & 0 & 0
\end{array}\right] \\
& =r\left[\begin{array}{cccc}
A_{1}-B_{1} X_{0} B_{1}^{*} & B_{1} & B_{1} & 0 \\
B_{1}^{*} & 0 & 0 & B^{*} \\
0 & B_{2} & 0 & 0 \\
0 & 0 & B_{3} & 0
\end{array}\right]-r(B)-r\left(B_{2}\right)-r\left(B_{3}\right) \\
& =r\left[\begin{array}{cccc}
A_{1} & B_{1} & B_{1} & B_{1} X_{0} B^{*} \\
B_{1}^{*} & 0 & 0 & B^{*} \\
0 & B_{2} & 0 & 0 \\
0 & 0 & B_{3} & 0
\end{array}\right]-r(B)-r\left(B_{2}\right)-r\left(B_{3}\right) \\
& =r\left[\begin{array}{ccccc}
A_{1} & B_{1} & B_{1} & 0 & 0 \\
B_{1}^{*} & 0 & 0 & B_{2}^{*} & B_{3}^{*} \\
0 & B_{2} & 0 & -A_{2} & 0 \\
0 & 0 & B_{3} & 0 & -A_{3}
\end{array}\right]-r(B)-r\left(B_{2}\right)-r\left(B_{3}\right) \\
& =r\left(Q_{1}\right)-r(B)-r\left(B_{2}\right)-r\left(B_{3}\right) \text {, } \\
& r\left[\begin{array}{cc}
A & G_{1} \\
G_{2} & 0
\end{array}\right]=r\left[\begin{array}{cc}
A_{1}-B_{1} X_{0} B_{1}^{*} & B_{1} \\
F_{B} B_{1}^{*} & 0
\end{array}\right]=r\left[\begin{array}{ccc}
A_{1} & B_{1} & 0 \\
B_{1}^{*} & 0 & B^{*}
\end{array}\right]-r(B) \\
& =r\left(P_{1}\right)-r(B) \text {, }
\end{aligned}
$$

$$
\begin{aligned}
i_{ \pm}\left[\begin{array}{cc}
A & G_{3} \\
G_{3}^{*} & 0
\end{array}\right]=i_{ \pm}\left[\begin{array}{ccc}
A_{1}-B_{1} X_{0} B_{1}^{*} & B_{1} F_{B_{2}} \\
F_{B_{2}} B_{1}^{*} & 0
\end{array}\right] \\
=i_{ \pm}\left[\begin{array}{ccc}
A_{1}-B_{1} X_{0} B_{1}^{*} & B_{1} & 0 \\
B_{1}^{*} & 0 & B_{2}^{*} \\
0 & B_{2} & 0
\end{array}\right]-r\left(B_{2}\right) \\
=i_{ \pm}\left[\begin{array}{ccc}
A_{1} & B_{1} & B_{1} X_{0} B_{2}^{*} / 2 \\
B_{1}^{*} & 0 & B_{2}^{*} \\
B_{1} X_{0} B_{2}^{*} / 2 & B_{2} & 0
\end{array}\right]-r\left(B_{2}\right) \\
=i_{ \pm}\left[\begin{array}{ccc}
A_{1} & B_{1} & 0 \\
B_{1}^{*} & 0 & B_{2}^{*} \\
0 & B_{2} & -A_{2}
\end{array}\right]-r\left(B_{2}\right)=i_{ \pm}\left(P_{2}\right)-r\left(B_{2}\right)
\end{aligned}
$$




$$
\begin{aligned}
& r\left[\begin{array}{ccc}
A & G_{3} & G_{4}^{*} \\
G_{3}^{*} & 0 & 0
\end{array}\right]=r\left[\begin{array}{ccc}
A_{1}-B_{1} X_{0} B_{1}^{*} & B_{1} F_{B_{2}} & B_{1} F_{B_{3}} \\
F_{B_{2}} B_{1}^{*} & 0 & 0
\end{array}\right] \\
& =r\left[\begin{array}{cccc}
A_{1}-B_{1} X_{0} B_{1}^{*} & B_{1} & B_{1} & 0 \\
B_{1}^{*} & 0 & 0 & B_{2}^{*} \\
0 & B_{2} & 0 & 0 \\
0 & 0 & B_{3} & 0
\end{array}\right]-2 r\left(B_{2}\right)-r\left(B_{3}\right) \\
& =r\left[\begin{array}{cccc}
A_{1} & B_{1} & B_{1} & B_{1} X_{0} B_{2}^{*} \\
B_{1}^{*} & 0 & 0 & B_{2}^{*} \\
0 & B_{2} & 0 & 0 \\
0 & 0 & B_{3} & 0
\end{array}\right]-2 r\left(B_{2}\right)-r\left(B_{3}\right) \\
& =r\left[\begin{array}{cccc}
A_{1} & B_{1} & B_{1} & 0 \\
B_{1}^{*} & 0 & 0 & B_{2}^{*} \\
0 & B_{2} & 0 & -A_{2} \\
0 & 0 & B_{3} & 0
\end{array}\right]-2 r\left(B_{2}\right)-r\left(B_{3}\right) \\
& =r\left(Q_{2}\right)-2 r\left(B_{2}\right)-r\left(B_{3}\right) \text {. }
\end{aligned}
$$

By a similar approach, we can obtain

$$
\begin{aligned}
& i_{ \pm}\left[\begin{array}{cc}
A & G_{4} \\
G_{4}^{*} & 0
\end{array}\right]=i_{ \pm}\left(P_{3}\right)-r\left(B_{3}\right), \\
& r\left[\begin{array}{ccc}
A & G_{3} & G_{4}^{*} \\
G_{4} & 0 & 0
\end{array}\right]=r\left(Q_{3}\right)-r\left(B_{2}\right)-2 r\left(B_{3}\right) .
\end{aligned}
$$

Substituting (3.36)-(3.42) into (3.32)-(3.35) yields (3.28)-(3.31).

Some direct consequences of the previous theorem are given below.

Corollary 3.4. Let $A_{i} \in \mathbb{C}_{\mathrm{H}}^{m_{i}}$ and $B_{i} \in \mathbb{C}^{m_{i} \times n}$ be given for $i=1,2,3$, and suppose that each pair of $B_{1} X B_{1}^{*}=A_{1}, B_{2} X B_{2}^{*}=A_{2}$ and $B_{3} X B_{3}^{*}=A_{3}$ have a common Hermitian solution. Also let $\mathcal{S}$ be defined in (1.2). Then,

$$
\begin{aligned}
\max _{X \in \mathcal{S}} r\left(A_{1}-B_{1} X B_{1}^{*}\right)=\min \left\{r\left(B_{1}\right), r\left(Q_{1}\right)-r\left[\begin{array}{l}
B_{2} \\
B_{3}
\end{array}\right]-r\left(B_{2}\right)-r\left(B_{3}\right),\right. \\
\left.2 r\left[\begin{array}{l}
B_{1} \\
B_{2}
\end{array}\right]-2 r\left(B_{2}\right), 2 r\left[\begin{array}{l}
B_{1} \\
B_{3}
\end{array}\right]-2 r\left(B_{3}\right)\right\}, \\
\min _{X \in \mathcal{S}} r\left(A_{1}-B_{1} X B_{1}^{*}\right)=2 r\left(Q_{1}\right)-2 r\left[\begin{array}{l}
B_{1} \\
B_{2} \\
B_{3}
\end{array}\right]-2 r\left[\begin{array}{cc}
B_{1} & B_{1} \\
B_{2} & 0 \\
0 & B_{3}
\end{array}\right], \\
\max _{X \in \mathcal{S}} i_{ \pm}\left(A_{1}-B_{1} X B_{1}^{*}\right)=\min \left\{r\left[\begin{array}{l}
B_{1} \\
B_{2}
\end{array}\right]-r\left(B_{2}\right), r\left[\begin{array}{c}
B_{1} \\
B_{3}
\end{array}\right]-r\left(B_{3}\right)\right\}, \\
\min _{X \in \mathcal{S}} i_{ \pm}\left(A_{1}-B_{1} X B_{1}^{*}\right)=r\left(Q_{1}\right)-r\left[\begin{array}{l}
B_{1} \\
B_{2} \\
B_{3}
\end{array}\right]-r\left[\begin{array}{cc}
B_{1} & B_{1} \\
B_{2} & 0 \\
0 & B_{3}
\end{array}\right],
\end{aligned}
$$

where $Q_{1}$ is of the form (3.26). 
Proof. Under the given conditions, the ranks and inertias of the block matrices in (3.25) and (3.27) are given by

$$
\begin{gathered}
r\left(P_{1}\right)=r\left(B_{1}\right)+r\left[\begin{array}{l}
B_{1} \\
B_{2} \\
B_{3}
\end{array}\right], r\left(P_{2}\right)=2 r\left[\begin{array}{l}
B_{1} \\
B_{2}
\end{array}\right], r\left(P_{3}\right)=2 r\left[\begin{array}{l}
B_{1} \\
B_{3}
\end{array}\right], \\
i_{ \pm}\left(P_{2}\right)=r\left[\begin{array}{l}
B_{1} \\
B_{2}
\end{array}\right], i_{ \pm}\left(P_{3}\right)=r\left[\begin{array}{l}
B_{1} \\
B_{3}
\end{array}\right], \\
r\left(Q_{2}\right)=r\left[\begin{array}{cc}
B_{1} & B_{1} \\
B_{2} & 0 \\
0 & B_{3}
\end{array}\right]+r\left[\begin{array}{l}
B_{1} \\
B_{2}
\end{array}\right], r\left(Q_{3}\right)=r\left[\begin{array}{cc}
B_{1} & B_{1} \\
B_{2} & 0 \\
0 & B_{3}
\end{array}\right]+r\left[\begin{array}{l}
B_{1} \\
B_{3}
\end{array}\right] .
\end{gathered}
$$

Hence (3.28)-(3.31) reduce to (3.43)-(3.46).

Corollary 3.5. Let $A_{i} \in \mathbb{C}_{\mathrm{H}}^{m_{i} \times m_{i}}$ and $B_{i} \in \mathbb{C}^{m_{i} \times n}$ be given for $i=1,2,3$, and suppose that each pair of the triple matrix equations

$$
B_{1} X B_{1}^{*}=A_{1}, \quad B_{2} X B_{2}^{*}=A_{2}, \quad B_{3} X B_{3}^{*}=A_{3}
$$

have a common Hermitian solution. Then, there exists a Hermitian $X$ such that (3.47) holds if and only if

$$
r\left[\begin{array}{ccccc}
A_{1} & 0 & 0 & B_{1} & B_{1} \\
0 & -A_{2} & 0 & B_{2} & 0 \\
0 & 0 & -A_{3} & 0 & B_{3} \\
B_{1}^{*} & B_{2}^{*} & B_{3}^{*} & 0 & 0
\end{array}\right]=r\left[\begin{array}{cc}
B_{1} & B_{1} \\
B_{2} & 0 \\
0 & B_{3}
\end{array}\right]+r\left[B_{1}^{*}, B_{2}^{*}, B_{3}^{*}\right] .
$$

Proof. It follows from (3.44).

A challenging open problem on the triple matrix equations in (3.47) is to give a parametric form for their general common Hermitian solution.

Setting $B_{1}=I_{n}$ in Theorem 3.3 may yield a group of results on the extremal ranks and inertias of $A_{1}-X$ subject to the set in (1.2). In particular, we have the following consequences.

Corollary 3.6. Let $A_{i} \in \mathbb{C}_{\mathrm{H}}^{m_{i}}$ and $B_{i} \in \mathbb{C}^{m_{i} \times n}$ be given for $i=2$, 3 , and assume that (3.24) has a common solution. Also let $\mathcal{S}$ be defined in (1.2). Then, the following hold.

(a) The global maximal rank of the matrices in (1.2) is

$$
\max _{X \in \mathcal{S}} r(X)=\min \left\{n, \quad s_{1}, \quad s_{2}, \quad s_{3}\right\},
$$

where

$$
\begin{gathered}
s_{1}=2 n+r\left[\begin{array}{ccc}
A_{2} & 0 & B_{2} \\
0 & A_{3} & B_{3}
\end{array}\right]-r\left[\begin{array}{l}
B_{2} \\
B_{3}
\end{array}\right]-r\left(B_{2}\right)-r\left(B_{3}\right), \\
s_{2}=2 n+r\left(A_{2}\right)-2 r\left(B_{2}\right), \quad s_{3}=2 n+r\left(A_{3}\right)-2 r\left(B_{3}\right) .
\end{gathered}
$$


(b) The global minimal rank of the matrices in (1.2) is

$$
\min _{X \in \mathcal{S}} r(X)=2 r\left[\begin{array}{ccc}
A_{2} & 0 & B_{2} \\
0 & A_{3} & B_{3}
\end{array}\right]+\max \left\{t_{1}, t_{2}, t_{3}, t_{4}\right\},
$$

where

$$
\begin{aligned}
& t_{1}=r\left(A_{2}\right)-2 r\left[\begin{array}{cc}
A_{2} & B_{2} \\
0 & B_{3}
\end{array}\right], \quad t_{2}=r\left(A_{3}\right)-2 r\left[\begin{array}{cc}
0 & B_{2} \\
A_{3} & B_{3}
\end{array}\right], \\
& t_{3}=i_{+}\left(A_{2}\right)+i_{-}\left(A_{3}\right)-r\left[\begin{array}{cc}
A_{2} & B_{2} \\
0 & B_{3}
\end{array}\right]-r\left[\begin{array}{cc}
0 & B_{2} \\
A_{3} & B_{3}
\end{array}\right], \\
& t_{4}=i_{-}\left(A_{2}\right)+i_{+}\left(A_{3}\right)-r\left[\begin{array}{cc}
A_{2} & B_{2} \\
0 & B_{3}
\end{array}\right]-r\left[\begin{array}{cc}
0 & B_{2} \\
A_{3} & B_{3}
\end{array}\right] .
\end{aligned}
$$

(c) The global maximal inertia of the matrices in (1.2) is

$$
\max _{X \in \mathcal{S}} i_{ \pm}(X)=\min \left\{n+i_{ \pm}\left(A_{2}\right)-r\left(B_{2}\right), \quad n+i_{ \pm}\left(A_{3}\right)-r\left(B_{3}\right)\right\} .
$$

(d) The global minimal inertia of the matrices in (1.2) is

$$
\begin{aligned}
\min _{X \in \mathcal{S}} i_{ \pm}(X)= & r\left[\begin{array}{ccc}
A_{2} & 0 & B_{2} \\
0 & A_{3} & B_{3}
\end{array}\right] \\
& +\max \left\{i_{ \pm}\left(A_{2}\right)-r\left[\begin{array}{cc}
A_{2} & B_{2} \\
0 & B_{3}
\end{array}\right], i_{ \pm}\left(A_{3}\right)-r\left[\begin{array}{cc}
0 & B_{2} \\
A_{3} & B_{3}
\end{array}\right]\right\} .
\end{aligned}
$$

In consequence, the following hold.

(e) Eq. (3.24) has a solution $X>0$ if and only if

$$
A_{2} \geqslant 0, \quad A_{3} \geqslant 0, \mathscr{R}\left(A_{2}\right)=\mathscr{R}\left(B_{2}\right), \quad \mathscr{R}\left(A_{3}\right)=\mathscr{R}\left(B_{3}\right) .
$$

(f) All solutions of (3.24) satisfy $X>0$ if and only if $A_{2} \geqslant 0, A_{3} \geqslant 0$ and one of

$$
r\left(A_{2}\right)=r\left(B_{2}\right)=n, \quad r\left(A_{3}\right)=r\left(B_{3}\right)=n .
$$

(g) Eq. (3.24) has a solution $X<0$ if and only if

$$
A_{2} \leqslant 0, \quad A_{3} \leqslant 0, \quad \mathscr{R}\left(A_{2}\right)=\mathscr{R}\left(B_{2}\right), \quad \mathscr{R}\left(A_{3}\right)=\mathscr{R}\left(B_{3}\right) .
$$

(h) All solutions of (3.24) satisfy $X<0$ if and only if $A_{2} \leqslant 0, A_{3} \leqslant 0$ and one of

$$
r\left(A_{2}\right)=r\left(B_{2}\right)=n, \quad r\left(A_{3}\right)=r\left(B_{3}\right)=n .
$$

(i) Eq. (3.24) has a solution $X \geqslant 0$ if and only if $A_{2} \geqslant 0, \quad A_{3} \geqslant 0, \quad \mathscr{R}\left[\begin{array}{c}A_{2} \\ 0\end{array}\right] \subseteq \mathscr{R}\left[\begin{array}{cc}0 & B_{2} \\ A_{3} & B_{3}\end{array}\right], \quad \mathscr{R}\left[\begin{array}{c}0 \\ A_{3}\end{array}\right] \subseteq \mathscr{R}\left[\begin{array}{cc}A_{2} & B_{2} \\ 0 & B_{3}\end{array}\right]$.

(j) All solutions of (3.24) satisfy $X \geqslant 0$ if and only if $A_{2} \geqslant 0, A_{3} \geqslant 0$ and one of

$$
r\left(B_{2}\right)=n, \quad r\left(B_{3}\right)=n
$$

(k) Eq. (3.24) has a solution $X \leqslant 0$ if and only if

$$
A_{2} \leqslant 0, \quad A_{3} \leqslant 0, \quad \mathscr{R}\left[\begin{array}{c}
A_{2} \\
0
\end{array}\right] \subseteq \mathscr{R}\left[\begin{array}{cc}
0 & B_{2} \\
A_{3} & B_{3}
\end{array}\right], \quad \mathscr{R}\left[\begin{array}{c}
0 \\
A_{3}
\end{array}\right] \subseteq \mathscr{R}\left[\begin{array}{cc}
A_{2} & B_{2} \\
0 & B_{3}
\end{array}\right] .
$$


(1) All solutions of (3.24) satisfy $X \leqslant 0$ if and only if $A_{2} \leqslant 0, A_{3} \leqslant 0$ and one of

$$
r\left(B_{2}\right)=n, \quad r\left(B_{3}\right)=n .
$$

Proof. Set $A_{1}=0$ and $B_{1}=I_{n}$ in Theorem 3.3 and simplifying, we obtain (a)-(d). Applying Lemma 2.1 to (3.49)-(3.52), we obtain the results in (e)-(l).

Corollary 3.6(e)-(l) give a set of analytical characterizations for the existence of definite common solutions of the two matrix equations in (3.24) by using some rank and range equalities and inequalities. These characterizations are simple and easy to understand in comparison with some known ambiguous conditions (see, e.g., $[12,40,41]$ ) on the existence of definite common solutions of (3.24).

Rewrite $B_{2} X B_{2}^{*}=A_{2}$ and $B_{3} X B_{3}^{*}=A_{3}$ as

$$
\left[B_{21}, B_{22}\right]\left[\begin{array}{cc}
X_{1} & X_{2} \\
X_{2}^{*} & X_{3}
\end{array}\right]\left[\begin{array}{l}
B_{21}^{*} \\
B_{22}^{*}
\end{array}\right]=A_{2}, \quad\left[B_{31}, B_{32}\right]\left[\begin{array}{cc}
X_{1} & X_{2} \\
X_{2}^{*} & X_{3}
\end{array}\right]\left[\begin{array}{l}
B_{31}^{*} \\
B_{32}^{*}
\end{array}\right]=A_{3},
$$

where $B_{i 1} \in \mathbb{C}^{m_{i} \times n_{1}}, B_{i 2} \in \mathbb{C}^{m_{i} \times n_{2}}, i=2,3, X_{1} \in \mathbb{C}_{\mathrm{H}}^{n_{1}}, X_{2} \in \mathbb{C}^{n_{1} \times n_{2}}$ and $X_{3} \in \mathbb{C}_{\mathrm{H}}^{n_{2}}$ with $n_{1}+n_{2}=n$. We next derive the extremal ranks and inertias of the submatrices $X_{1}$ and $X_{3}$ in a Hermitian solution of (3.53). Note that $X_{1}, X_{2}, X_{3}$ in (3.53) can be rewritten as

$$
X_{1}=P_{1} X P_{1}^{*}, \quad X_{2}=P_{1} X P_{2}^{*}, \quad X_{3}=P_{2} X P_{2}^{*},
$$

where $P_{1}=\left[I_{n_{1}}, 0\right]$ and $P_{2}=\left[0, I_{n_{2}}\right]$. For convenience, we adopt the following notation for the collections of the submatrices $X_{1}$ and $X_{3}$ in (3.53):

$$
\begin{aligned}
& \mathcal{S}_{1}=\left\{X_{1}=P_{1} X P_{1}^{*} \mid B_{2} X B_{2}^{*}=A_{2}, B_{3} X B_{3}^{*}=A_{3}, X=X^{*}\right\}, \\
& \mathcal{S}_{3}=\left\{X_{3}=P_{2} X P_{2}^{*} \mid B_{2} X B_{2}^{*}=A_{2}, B_{3} X B_{3}^{*}=A_{3}, X=X^{*}\right\} .
\end{aligned}
$$

The global maximal and minimal ranks and inertias of the submatrices $X_{1}$ and $X_{3}$ in (3.53) can easily be derived from Theorem 3.3. The details are omitted.

If each of the triple matrix equations in (1.8) is not consistent, people may alternatively seek its common approximation solutions under various given optimal criteria. One of the most useful approximation solutions of $B X B^{*}=A$ is the well-known least-squares Hermitian solution, which is defined to be a Hermitian matrix $X$ that minimizes the objective function:

$$
\left\|A-B X B^{*}\right\|^{2}=\operatorname{tr}\left[\left(A-B X B^{*}\right)\left(A-B X B^{*}\right)^{*}\right] .
$$

The normal equation corresponding to the norm minimization problem is given by

$$
B^{*} B X B^{*} B=B^{*} A B
$$

This equation is always consistent. Concerning the common least-squares Hermitian solution of (1.8), we have the following result.

Corollary 3.7. Let $A_{i} \in \mathbb{C}_{\mathrm{H}}^{m_{i}}$ and $B_{i} \in \mathbb{C}^{m_{i} \times n}$ be given for $i=1,2,3$. Then, there exists an $X \in \mathbb{C}_{\mathrm{H}}^{n}$ such that

$$
\left\|A_{i}-B_{i} X B_{i}^{*}\right\|=\min , \quad i=1,2,3,
$$


hold simultaneously, if and only if

$$
\begin{aligned}
& r\left[\begin{array}{ccc}
B_{i}^{*} A_{i} B_{i} & 0 & B_{i}^{*} B_{i} \\
0 & -B_{j}^{*} A_{j} B_{j} & B_{j}^{*} B_{j} \\
B_{i}^{*} B_{i} & B_{j}^{*} B_{j} & 0
\end{array}\right]=2 r\left[\begin{array}{c}
B_{i} \\
B_{j}
\end{array}\right], \quad i \neq j, i, j=1,2,3, \\
& r\left[\begin{array}{ccccc}
B_{1}^{*} A_{1} B_{1} & 0 & 0 & B_{1}^{*} B_{1} & B_{1}^{*} B_{1} \\
0 & -B_{2}^{*} A_{2} B_{2} & 0 & B_{2}^{*} B_{2} & 0 \\
0 & 0 & -B_{3}^{*} A_{3} B_{3} & 0 & B_{3}^{*} B_{3} \\
B_{1}^{*} B_{1} & B_{2}^{*} B_{2} & B_{3}^{*} B_{3} & 0 & 0
\end{array}\right]=r\left[\begin{array}{cc}
B_{1} & B_{1} \\
B_{2} & 0 \\
0 & B_{3}
\end{array}\right]+r\left[\begin{array}{l}
B_{1} \\
B_{2} \\
B_{3}
\end{array}\right] .
\end{aligned}
$$

Proof. It follows from Lemma 3.1, Corollary 3.5 and (3.58).

4. The extremal Ranks and inertias of $A_{1}-B_{1} X B_{1}^{*}$ Subject to the Hermitian solutions of $B_{4} X=A_{4}$

Although $B_{4} X=A_{4}$ in (1.3) is not symmetric in form, it may have a Hermitian solution, as shown in Lemma 2.6. So that the global extremal ranks and inertias of $A_{1}-B_{1} X B_{1}^{*}$ subject to the Hermitian solution or positive semi-definite solution of $B_{4} X=A_{4}$ can also be derived.

Theorem 4.1. Assume that the matrix equation $B_{4} X=A_{4}$ in (1.3) has a Hermitian solution, i.e., $\mathscr{R}\left(A_{4}\right) \subseteq \mathscr{R}\left(B_{4}\right)$ and $A_{4} B_{4}^{*}=B_{4} A_{4}^{*}, \mathcal{T}$ is defined in (1.3), and let

$$
M=\left[\begin{array}{cc}
A_{1} & B_{1} \\
A_{4} B_{1}^{*} & B_{4}
\end{array}\right], N=\left[\begin{array}{ccc}
A_{1} & B_{1} & 0 \\
B_{1}^{*} & 0 & B_{4}^{*} \\
0 & B_{4} & -A_{4} B_{4}^{*}
\end{array}\right]
$$

Then,

$$
\begin{gathered}
\max _{X \in \mathcal{T}} r\left(A_{1}-B_{1} X B_{1}^{*}\right)=r(M)-r\left(B_{4}\right), \\
\min _{X \in \mathcal{T}} r\left(A_{1}-B_{1} X B_{1}^{*}\right)=2 r(M)-r(N), \\
\max _{X \in \mathcal{T}} i_{ \pm}\left(A_{1}-B_{1} X B_{1}^{*}\right)=i_{ \pm}(N)-r\left(B_{4}\right), \\
\min _{X \in \mathcal{T}} i_{ \pm}\left(A_{1}-B_{1} X B_{1}^{*}\right)=r(M)-i_{\mp}(N) .
\end{gathered}
$$

In consequences, the following hold.

(a) $B_{4} X=A_{4}$ has a solution $X \in \mathbb{C}_{\mathrm{H}}^{n}$ such that $A_{1}-B_{1} X B_{1}^{*}$ is nonsingular if and only if $r(M)=r\left(B_{4}\right)+m_{1}$.

(b) $A_{1}-B_{1} X B_{1}^{*}$ is nonsingular for all Hermitian solution of $B_{4} X=A_{4}$ if and only if $2 r(M)=r(N)+m_{1}$.

(c) The pair of matrix equations $B_{1} X B_{1}^{*}=A_{1}$ and $B_{4} X=A_{4}$ have a common Hermitian solution if and only if $\mathscr{R}\left[\begin{array}{c}A_{1} \\ A_{4} B_{1}^{*}\end{array}\right] \subseteq \mathscr{R}\left[\begin{array}{c}B_{1} \\ B_{4}\end{array}\right]$.

(d) $B_{1} X B_{1}^{*}=A_{1}$ holds for all Hermitian solutions of $B_{4} X=A_{4}$ if and only if $r(M)=r\left(B_{4}\right)$.

(e) $B_{4} X=A_{4}$ has a solution $X \in \mathbb{C}_{\mathrm{H}}^{n}$ such that $A_{1}-B_{1} X B_{1}^{*}>0\left(A_{1}-\right.$ $\left.B_{1} X B_{1}^{*}<0\right)$ if and only if $i_{+}(N)=r\left(B_{4}\right)+m_{1} \quad\left(i_{-}(N)=r\left(B_{4}\right)+m_{1}\right)$. 
(f) $A_{1}-B_{1} X B_{1}^{*}>0\left(A_{1}-B_{1} X B_{1}^{*}<0\right)$ holds for all Hermitian solutions of $B_{4} X=A_{4}$ if and only if $r(M)=i_{-}(N)+m_{1}\left(r(M)=i_{+}(N)+m_{1}\right)$.

(g) $B_{4} X=A_{4}$ has a solution $X \in \mathbb{C}_{\mathrm{H}}^{n}$ such that $A_{1}-B_{1} X B_{1}^{*} \geqslant 0\left(A_{1}-\right.$ $\left.B_{1} X B_{1}^{*} \leqslant 0\right)$ if and only if $r(M)=i_{+}(N) \quad\left(r(M)=i_{-}(N)\right)$.

(f) $A_{1}-B_{1} X B_{1}^{*} \geqslant 0\left(A_{1}-B_{1} X B_{1}^{*} \leqslant 0\right)$ holds for all Hermitian solutions of $B_{4} X=A_{4}$ if and only if $i_{-}(N)=r\left(B_{4}\right)\left(i_{+}(N)=r\left(B_{4}\right)\right)$.

Proof. From Lemma 2.6(a), the general Hermitian solution of $B_{4} X=A_{4}$ can be written as

$$
X=B_{4}^{\dagger} A_{4}+\left(B_{4}^{\dagger} A_{4}\right)^{*}-B_{4}^{\dagger} A_{4} B_{4}^{\dagger} B_{4}+F_{B_{4}} W F_{B_{4}}
$$

where $W \in \mathbb{C}_{\mathrm{H}}^{n}$ is arbitrary. Substituting (4.6) into $A_{1}-B_{1} X B_{1}^{*}$ gives

$$
A_{1}-B_{1} X B_{1}^{*}=G-B_{1} F_{B_{4}} W F_{B_{4}} B_{1}^{*}
$$

where $G=A_{1}-B_{1} B_{4}^{\dagger} A_{4} B_{1}^{*}-B_{1}\left(B_{4}^{\dagger} A_{4}\right)^{*} B_{1}^{*}+B_{1} B_{4}^{\dagger} A_{4} B_{4}^{\dagger} B_{4} B_{1}^{*}$. Applying (2.25)(2.28) to (4.7) yields

$$
\begin{aligned}
\max _{X \in \mathcal{T}} r\left(A_{1}-B_{1} X B_{1}^{*}\right) & =\max _{W \in \mathbb{C}_{\mathrm{H}}^{n}} r\left(G-B_{1} F_{B_{4}} W F_{B_{4}} B_{1}^{*}\right)=r\left[G, B_{1} F_{B_{4}}\right], \\
\min _{X \in \mathcal{T}} r\left(A_{1}-B_{1} X B_{1}^{*}\right) & =\min _{W \in \mathbb{C}_{\mathrm{H}}^{n}} r\left(G-B_{1} F_{B_{4}} W F_{B_{4}} B_{1}^{*}\right) \\
& =2 r\left[G, B_{1} F_{B_{4}}\right]-r\left[\begin{array}{cc}
G & B_{1} F_{B_{4}} \\
F_{B_{4}} B_{1}^{*} & 0
\end{array}\right],
\end{aligned}
$$

$$
\begin{aligned}
\max _{X \in \mathcal{T}} i_{ \pm}\left(A_{1}-B_{1} X B_{1}^{*}\right) & =\max _{W \in \mathbb{C}_{\mathrm{H}}^{n}} r\left(G-B_{1} F_{B_{4}} W F_{B_{4}} B_{1}^{*}\right) \\
& =i_{ \pm}\left[\begin{array}{cc}
G & B_{1} F_{B_{4}} \\
F_{B_{4}} B_{1}^{*} & 0
\end{array}\right], \\
\min _{X \in \mathcal{T}} i_{ \pm}\left(A_{1}-B_{1} X B_{1}^{*}\right) & =\min _{W \in \mathbb{C}_{\mathrm{H}}^{n}} r\left(G-B_{1} F_{B_{4}} W F_{B_{4}} B_{1}^{*}\right) \\
& =r\left[G, B_{1} F_{B_{4}}\right]-i_{\mp}\left[\begin{array}{cc}
G & B_{1} F_{B_{4}} \\
F_{B_{4}} B_{1}^{*} & 0
\end{array}\right] .
\end{aligned}
$$

It is easy to verify that under $B_{4} B_{4}^{\dagger} A_{4}=A_{4}$, the equality

$$
B_{4}\left(B_{4}^{\dagger} A_{4}\right)^{*}=B_{4} A_{4}^{*}\left(B_{4}^{\dagger}\right)^{*}=A_{4} B_{4}^{*}\left(B_{4}^{\dagger}\right)^{*}=A_{4} B_{4}^{\dagger} B_{4}
$$

holds. In this case, applying (2.5), (2.15) and (2.16) to (4.8)-(4.11) and simplifying by elementary matrix operations and congruence matrix operations, we 
obtain

$$
\begin{aligned}
& r\left[G, B_{1} F_{B_{4}}\right] \\
& =r\left[\begin{array}{cc}
A_{1}-B_{1} B_{4}^{\dagger} A_{4} B_{1}^{*}-B_{1}\left(B_{4}^{\dagger} A_{4}\right)^{*} B_{1}^{*}+B_{1} B_{4}^{\dagger} A_{4} B_{4}^{\dagger} B_{4} B_{1}^{*} & B_{1} \\
0 & B_{4}
\end{array}\right]-r\left(B_{4}\right) \\
& =r\left[\begin{array}{cc}
A_{1} & B_{1} \\
A_{4} B_{1}^{*}+B_{4}\left(B_{4}^{\dagger} A_{4}\right)^{*} B_{1}^{*}-A_{4} B_{4}^{\dagger} B_{4} B_{1}^{*} & B_{4}
\end{array}\right]-r\left(B_{4}\right) \\
& =r\left[\begin{array}{cc}
A_{1} & B_{1} \\
A_{4} B_{1}^{*} & B_{4}
\end{array}\right]-r\left(B_{4}\right)=r(M)-r\left(B_{4}\right), \\
& i_{ \pm}\left[\begin{array}{cc}
G & B_{1} F_{B_{4}} \\
F_{B_{4}} B_{1}^{*} & 0
\end{array}\right] \\
& =i_{ \pm}\left[\begin{array}{ccc}
A_{1}-B_{1} B_{4}^{\dagger} A_{4} B_{1}^{*}-B_{1}\left(B_{4}^{\dagger} A_{4}\right)^{*} B_{1}^{*}+B_{1} B_{4}^{\dagger} A_{4} B_{4}^{\dagger} B_{4} B_{1}^{*} & B_{1} & 0 \\
B_{1}^{*} & 0 & B_{4}^{*} \\
0 & B_{4} & 0
\end{array}\right]-r\left(B_{4}\right) \\
& =i_{ \pm}\left[\begin{array}{ccc}
A_{1} & B_{1} & \frac{1}{2} B_{1} B_{4}^{\dagger} A_{4} B_{4}^{*}+\frac{1}{2} B_{1} A_{4}^{*}-\frac{1}{2} B_{1} B_{4}^{\dagger} A_{4} B_{4}^{*} \\
B_{1}^{*} & 0 & B_{4}^{*} \\
\frac{1}{2} A_{4} B_{1}^{*}+\frac{1}{2} B_{4} B_{1}\left(B_{4}^{\dagger} A_{4}\right)^{*} B_{1}^{*}-\frac{1}{2} A_{4} B_{4}^{\dagger} B_{4} B_{1}^{*} & B_{4} & 0
\end{array}\right] \\
& -r\left(B_{4}\right) \\
& =i_{ \pm}\left[\begin{array}{ccc}
A_{1} & B_{1} & \frac{1}{2} B_{1} A_{4}^{*} \\
B_{1}^{*} & 0 & B_{4}^{*} \\
\frac{1}{2} A_{4} B_{1}^{*} & B_{4} & 0
\end{array}\right]-r\left(B_{4}\right)=i_{ \pm}\left[\begin{array}{ccc}
A_{1} & B_{1} & 0 \\
B_{1}^{*} & 0 & B_{4}^{*} \\
0 & B_{4} & -A_{4} B_{4}^{*}
\end{array}\right]-r\left(B_{4}\right) \\
& =i_{ \pm}(N)-r\left(B_{4}\right) \text {. }
\end{aligned}
$$

Substituting (4.12) and (4.13) into (4.8)-(4.11) yields (4.2)-(4.5). Applying Lemma 2.1 to $(4.2)-(4.5)$ yields (a)-(f).

Theorem 4.2. Assume that the matrix equation $B_{4} X=A_{4}$ in (1.3) has a positive semi-definite solution, i.e., $\mathscr{R}\left(A_{4}\right) \subseteq \mathscr{R}\left(B_{4}\right), A_{4} B_{4}^{*} \geqslant 0$ and $r\left(A_{4} B_{4}^{*}\right)=r\left(A_{4}\right)$, and let

$$
\begin{aligned}
& \mathcal{T}=\left\{0 \leqslant X \in \mathbb{C}_{\mathrm{H}}^{n} \mid A_{4} X=B_{4}\right\}, M_{1}=\left[\begin{array}{cc}
A_{1} & B_{1} \\
A_{4} B_{1}^{*} & B_{4}
\end{array}\right], \\
& M_{2}=\left[\begin{array}{cc}
A_{1} & B_{1} A_{4}^{*} \\
A_{4} B_{1}^{*} & A_{4} B_{4}^{*}
\end{array}\right], N=\left[\begin{array}{ccc}
A_{1} & B_{1} & 0 \\
B_{1}^{*} & 0 & B_{4}^{*} \\
0 & B_{4} & -A_{4} B_{4}^{*}
\end{array}\right] .
\end{aligned}
$$


Then,

$$
\begin{aligned}
\max _{X \in \mathcal{T}} r\left(A_{1}-B_{1} X B_{1}^{*}\right) & =r\left(M_{1}\right)-r\left(B_{4}\right), \\
\min _{X \in \mathcal{T}} r\left(A_{1}-B_{1} X B_{1}^{*}\right) & =r\left(M_{1}\right)+i_{-}\left(M_{2}\right)-i_{-}(N), \\
\max _{X \in \mathcal{T}} i_{+}\left(A_{1}-B_{1} X B_{1}^{*}\right) & =i_{+}\left(M_{2}\right)-r\left(A_{4}\right), \\
\min _{X \in \mathcal{T}} i_{+}\left(A_{1}-B_{1} X B_{1}^{*}\right) & =r\left(M_{1}\right)-i_{-}(N), \\
\max _{X \in \mathcal{T}} i_{-}\left(A_{1}-B_{1} X B_{1}^{*}\right) & =i_{-}(N)-r\left(A_{4}\right), \\
\min _{X \in \mathcal{T}} i_{-}\left(A_{1}-B_{1} X B_{1}^{*}\right) & =i_{-}\left(M_{2}\right) .
\end{aligned}
$$

In consequences, the following hold.

(a) $B_{4} X=A_{4}$ has a positive semi-definite solution such that $A_{1}-B_{1} X B_{1}^{*}$ is nonsingular if and only if $r\left(M_{1}\right)=r\left(B_{4}\right)+m_{1}$.

(b) $A_{1}-B_{1} X B_{1}^{*}$ is nonsingular for all positive semi-definite solution of $B_{4} X=$ $A_{4}$ if and only if $r\left(M_{1}\right)+i_{-}\left(M_{2}\right)=i_{-}(N)+m_{1}$.

(c) The pair of matrix equations $B_{1} X B_{1}^{*}=A_{1}$ and $B_{4} X=A_{4}$ have a common positive semi-definite solution if and only if $r\left(M_{1}\right)+i_{-}\left(M_{2}\right)=i_{-}(N)$.

(d) $B_{1} X B_{1}^{*}=A_{1}$ holds for all positive semi-definite solutions of $B_{4} X=A_{4}$ if and only if $r(M)=r\left(B_{4}\right)$.

(e) $B_{4} X=A_{4}$ has a solution $0 \leqslant X \in \mathbb{C}_{\mathrm{H}}^{n}$ such that $A_{1}-B_{1} X B_{1}^{*}>0$ if and only if $i_{+}\left(M_{2}\right)=r\left(A_{4}\right)+m_{1}$.

(f) $A_{1}-B_{1} X B_{1}^{*}>0$ holds for all positive semi-definite solutions of $B_{4} X=A_{4}$ if and only if $r\left(M_{1}\right)=i_{-}(N)+m_{1}$.

(g) $B_{4} X=A_{4}$ has a solution $0 \leqslant X \in \mathbb{C}_{\mathrm{H}}^{n}$ such that $A_{1}-B_{1} X B_{1}^{*}<0$ if and only if $i_{-}(N)=r\left(A_{4}\right)+m_{1}$.

(h) $A_{1}-B_{1} X B_{1}^{*}<0$ holds for all positive semi-definite solutions of $B_{4} X=A_{4}$ if and only if $i_{-}\left(M_{2}\right)=m_{1}$.

(i) $B_{4} X=A_{4}$ has a solution $0 \leqslant X \in \mathbb{C}_{\mathrm{H}}^{n}$ such that $A_{1}-B_{1} X B_{1}^{*} \geqslant 0$ if and only if $M_{2} \geqslant 0$.

(j) $A_{1}-B_{1} X B_{1}^{*} \geqslant 0$ holds for all positive semi-definite solutions of $B_{4} X=A_{4}$ if and only if $i_{-}(N)=r\left(A_{4}\right)$.

(k) $B_{4} X=A_{4}$ has a solution $0 \leqslant X \in \mathbb{C}_{\mathrm{H}}^{n}$ such that $A_{1}-B_{1} X B_{1}^{*} \leqslant 0$ if and only if $r\left(M_{1}\right)=i_{-}(N)$.

(l) $A_{1}-B_{1} X B_{1}^{*} \leqslant 0$ holds for all positive semi-definite solutions of $B_{4} X=A_{4}$ if and only if $i_{+}\left(M_{2}\right)=r\left(A_{4}\right)$.

Proof. From Lemma 2.6(b), the general positive semi-definite solution of $B_{4} X=$ $A_{4}$ can be written as

$$
X=A_{4}^{*}\left(A_{4} B_{4}^{*}\right)^{\dagger} A_{4}+F_{B_{4}} W F_{B_{4}}
$$

where $0 \leqslant W \in \mathbb{C}_{\mathrm{H}}^{n}$ is arbitrary. Substituting (4.22) into $A_{1}-B_{1} X B_{1}^{*}$ gives

$$
A_{1}-B_{1} X B_{1}^{*}=G-B_{1} F_{B_{4}} W F_{B_{4}} B_{1}^{*},
$$


where $G=A_{1}-B_{1} A_{4}^{*}\left(A_{4} B_{4}^{*}\right)^{\dagger} A_{4} B_{1}^{*}$. Applying (2.37)-(2.40) to (4.23) yields

$$
\begin{aligned}
& \max _{X \in \mathcal{T}} r\left(A_{1}-B_{1} X B_{1}^{*}\right)=\max _{0 \leqslant W \in \mathbb{C}_{\mathrm{H}}^{n}} r\left(G-B_{1} F_{B_{4}} W F_{B_{4}} B_{1}^{*}\right) \\
& =r\left[G, B_{1} F_{B_{4}}\right] \\
& \min _{X \in \mathcal{T}} r\left(A_{1}-B_{1} X B_{1}^{*}\right)=\min _{0 \leqslant W \in \mathbb{C}_{\mathrm{H}}^{n}} r\left(G-B_{1} F_{B_{4}} W F_{B_{4}} B_{1}^{*}\right) \\
& =i_{-}(G)+r\left[G, B_{1} F_{B_{4}}\right]-i_{-}\left[\begin{array}{cc}
G & B_{1} F_{B_{4}} \\
F_{B_{4}} B_{1}^{*} & 0
\end{array}\right], \\
& \max _{X \in \mathcal{T}} i_{+}\left(A_{1}-B_{1} X B_{1}^{*}\right)=\max _{0 \leqslant W \in \mathbb{C}_{\mathrm{H}}^{n}} r\left(G-B_{1} F_{B_{4}} W F_{B_{4}} B_{1}^{*}\right)=i_{+}(G), \\
& \min _{X \in \mathcal{T}} i_{+}\left(A_{1}-B_{1} X B_{1}^{*}\right)=\max _{0 \leqslant W \in \mathbb{C}_{\mathrm{H}}^{n}} i_{+}\left(G-B_{1} F_{B_{4}} W F_{B_{4}} B_{1}^{*}\right) \\
& =r\left[G, B_{1} F_{B_{4}}\right]-i_{-}\left[\begin{array}{cc}
G & B_{1} F_{B_{4}} \\
F_{B_{4}} B_{1}^{*} & 0
\end{array}\right], \\
& \max _{X \in \mathcal{T}} i_{-}\left(A_{1}-B_{1} X B_{1}^{*}\right)=\max _{0 \leqslant W \in \mathbb{C}_{\mathrm{H}}^{n}} i_{-}\left(G-B_{1} F_{B_{4}} W F_{B_{4}} B_{1}^{*}\right) \\
& =i_{-}\left[\begin{array}{cc}
G & B_{1} F_{B_{4}} \\
F_{B_{4}} B_{1}^{*} & 0
\end{array}\right], \\
& \min _{X \in \mathcal{T}} i_{-}\left(A_{1}-B_{1} X B_{1}^{*}\right)=\min _{0 \leqslant W \in \mathbb{C}_{\mathrm{H}}^{n}} i_{-}\left(G-B_{1} F_{B_{4}} W F_{B_{4}} B_{1}^{*}\right)=i_{-}(G) .
\end{aligned}
$$

Applying (2.4)-(2.6), (2.15) and (2.16) and simplifying, we obtain

$$
\begin{aligned}
& r\left[G, B_{1} F_{B_{4}}\right]=r\left[\begin{array}{ccc}
A_{1}-B_{1} A_{4}^{*}\left(A_{4} B_{4}^{*}\right)^{\dagger} A_{4} B_{1}^{*} & B_{1} \\
0 & B_{4}
\end{array}\right]-r\left(B_{4}\right) \\
&=r {\left[\begin{array}{cc}
A_{1} & B_{1} \\
A_{4} B_{1}^{*} & B_{4}
\end{array}\right]-r\left(B_{4}\right), } \\
& i_{ \pm}\left[\begin{array}{cc}
G & B_{1} F_{B_{4}} \\
F_{B_{4}} B_{1}^{*} & 0
\end{array}\right]=i_{ \pm}\left[\begin{array}{ccc}
A_{1}-B_{1} A_{4}^{*}\left(A_{4} B_{4}^{*}\right)^{\dagger} A_{4} B_{1}^{*} & B_{1} & 0 \\
B_{1}^{*} & 0 & B_{4}^{*} \\
0 & B_{4} & 0
\end{array}\right]-r\left(B_{4}\right) \\
&=i_{ \pm}\left[\begin{array}{ccc}
A_{1} & B_{1} & \frac{1}{2} B_{1} A_{4}^{*} \\
B_{1}^{*} & 0 & B_{4}^{*} \\
\frac{1}{2} A_{4} B_{1}^{*} & B_{4} & 0
\end{array}\right]-r\left(B_{4}\right) \\
&=i_{ \pm}\left[\begin{array}{ccc}
A_{1} & B_{1} & 0 \\
B_{1}^{*} & 0 & B_{4}^{*} \\
0 & B_{4} & -A_{4} B_{4}^{*}
\end{array}\right]-r\left(B_{4}\right) \\
& i_{ \pm}(G)=i_{ \pm}\left[\begin{array}{cc}
A_{1}-B_{1} A_{4}^{*}\left(A_{4} B_{4}^{*}\right)^{\dagger} A_{4} B_{1}^{*}
\end{array}\right] \\
&=i_{ \pm}\left[\begin{array}{cc}
A_{1} & B_{1} A_{4}^{*} \\
A_{4} B_{1}^{*} & A_{4} B_{4}^{*}
\end{array}\right]-i_{ \pm}\left(A_{4} B_{4}^{*}\right) .
\end{aligned}
$$

Substituting (4.30)-(4.32) into (4.24)-(4.29) yields (4.16)-(4.21). Applying Lemma 2.1 to $(4.2)-(4.5)$ yields (a)-(l).

Corollary 4.3. Assume that the matrix equation $A X=B$ has a Hermitian solution, i.e., $\mathscr{R}(B) \subseteq \mathscr{R}(A)$ and $A B^{*}=B A^{*}$, where $A, B \in \mathbb{C}^{m \times n}$ are given, let 
$P \in \mathbb{C}_{\mathrm{H}}^{n}$ and define $\mathcal{T}=\left\{X \in \mathbb{C}_{\mathrm{H}}^{n} \mid A X=B\right\}$. Then,

$$
\begin{aligned}
\max _{X \in \mathcal{T}} r(X-P) & =r(B-A P)-r(A)+n, \\
\min _{X \in \mathcal{T}} r(X-P) & =2 r(B-A P)-r\left(B A^{*}-A P A^{*}\right), \\
\max _{X \in \mathcal{T}} i_{ \pm}(X-P) & =i_{ \pm}\left(B A^{*}-A P A^{*}\right)-r(A)+n, \\
\min _{X \in \mathcal{T}} i_{ \pm}(X-P) & =r(B-A P)-i_{\mp}\left(B A^{*}-A P A^{*}\right) .
\end{aligned}
$$

In consequence, the following hold.

(a) There exists an $X \in \mathcal{T}$ such that $X-P$ is nonsingular if and only if

$$
\mathscr{R}(A P-B)=\mathscr{R}(A) .
$$

(b) $X-P$ is nonsingular for all $X \in \mathcal{T}$ if and only if

$$
2 r(B-A P)=r\left(B A^{*}-A P A^{*}\right)+n .
$$

(c) There exists an $X \in \mathcal{T}$ such that $X>P(X<P)$ holds if and only if

$$
\begin{gathered}
\mathscr{R}\left(B A^{*}-A P A^{*}\right)=\mathscr{R}(A) \text { and } B A^{*} \geqslant A P A^{*} \\
\left(\mathscr{R}\left(B A^{*}-A P A^{*}\right)=\mathscr{R}(A) \text { and } B A^{*} \leqslant A P A^{*}\right) .
\end{gathered}
$$

(d) $X>P(X<P)$ holds for all $X \in \mathcal{T}$ if and only if $r(B-A P)=n$ and $B A^{*} \geqslant A P A^{*} \quad\left(r(B-A P)=n\right.$ and $\left.A B^{*} \leqslant A P A^{*}\right)$.

(e) There exists an $X \in \mathcal{T}$ such that $X \geqslant P(X \leqslant P)$ holds if and only if

$$
\begin{gathered}
\mathscr{R}(B-A P)=\mathscr{R}\left(B A^{*}-A P A^{*}\right) \text { and } B A^{*} \geqslant A P A^{*} \\
\left(\mathscr{R}(B-A P)=\mathscr{R}\left(B A^{*}-A P A^{*}\right) \text { and } B A^{*} \leqslant A P A^{*}\right) .
\end{gathered}
$$

(f) $X \geqslant P(X \leqslant P)$ holds for all $X \in \mathcal{T}$ if and only if

$$
B A^{*} \geqslant A P A^{*} \text { and } r(A)=n \quad\left(B A^{*} \leqslant A P A^{*} \text { and } r(A)=n\right) \text {. }
$$

Corollary 4.4. Assume that the matrix equation $A X=B$ has a Hermitian solution $X \geqslant 0$, where $A, B \in \mathbb{C}^{m \times n}$ are given, let $0 \leqslant P \in \mathbb{C}_{\mathrm{H}}^{n}$ and define

$$
\mathcal{T}=\left\{0 \leqslant X \in \mathbb{C}_{\mathrm{H}}^{n} \mid A X=B\right\}, \quad M=\left[\begin{array}{cc}
B A^{*} & B \\
B^{*} & P
\end{array}\right] \text {. }
$$

Then,

$$
\begin{aligned}
\max _{X \in \mathcal{T}} r(X-P) & =r(B-A P)-r(A)+n, \\
\min _{X \in \mathcal{T}} r(X-P) & =i_{-}(M)+r(B-A P)-i_{+}\left(B A^{*}-A P A^{*}\right), \\
\max _{X \in \mathcal{T}} i_{+}(X-P) & =i_{+}\left(B A^{*}-A P A^{*}\right)-r(A)+n, \\
\min _{X \in \mathcal{T}} i_{+}(X-P) & =i_{-}(M), \\
\max _{X \in \mathcal{T}} i_{-}(X-P) & =i_{+}(M)-r(B), \\
\min _{X \in \mathcal{T}} i_{-}(X-P) & =r(B-A P)-i_{+}\left(B A^{*}-A P A^{*}\right) .
\end{aligned}
$$

In consequence, the following hold. 
(a) There exists an $X \in \mathcal{T}$ such that $X-P$ is nonsingular if and only if $\mathscr{R}(B-A P)=\mathscr{R}(A)$.

(b) $X-P$ is nonsingular for all $X \in \mathcal{T}$ if and only if $i_{-}(M)+r(B-A P)=$ $i_{+}\left(B A^{*}-A P A^{*}\right)+n$.

(c) There exists an $X \in \mathcal{T}$ such that $X>P$ holds if and only if $\mathscr{R}\left(B A^{*}-\right.$ $\left.A P A^{*}\right)=\mathscr{R}(A)$ and $B A^{*} \geqslant A P A^{*}$.

(d) $X>P$ holds for all $X \in \mathcal{T}$ if and only if $i_{-}(M)=r(A)$.

(e) There exists an $X \in \mathcal{T}$ such that $X<P$ holds if and only if $i_{-}(M)=$ $r(B)+n$.

(f) $X<P$ holds for all $X \in \mathcal{T}$ if and only if $r(B-A P)=n$ and $B A^{*} \leqslant$ $A P A^{*}$.

(g) There exists an $X \in \mathcal{T}$ such that $X \geqslant P$ if and only if $\mathscr{R}(B-A P)=$ $\mathscr{R}\left(B A^{*}-A P A^{*}\right)$ and $B A^{*} \geqslant A P A^{*}$.

(h) $X \geqslant P$ holds for all $X \in \mathcal{T}$ if and only if $i_{-}(M)=r(B)$.

(i) There exists an $X \in \mathcal{T}$ such that $X \leqslant P$ if and only if $M \geqslant 0$.

(j) $X \leqslant P$ holds for all $X \in \mathcal{T}$ if and only if $i_{+}\left(B A^{*}-A P A^{*}\right)=n-r(A)$.

Corollary 4.5. Assume that the matrix equation $A X=B$ has a Hermitian solution, i.e., $\mathscr{R}(B) \subseteq \mathscr{R}(A)$ and $A B^{*}=B A^{*}$, where $A, B \in \mathbb{C}^{m \times n}$ are given. Then,

$$
\begin{gathered}
\max _{A X=B, X \in \mathbb{C}_{\mathrm{H}}^{n}} r(X)=n+r(B)-r(A), \\
\min _{A X=B, X \in \mathbb{C}_{\mathrm{H}}^{n}} r(X)=2 r(B)-r\left(A B^{*}\right), \\
\max _{A X=B, X \in \mathbb{C}_{\mathrm{H}}^{n}} i_{ \pm}(X)=n+i_{ \pm}\left(A B^{*}\right)-r(A), \\
\min _{A X=B, X \in \mathbb{C}_{\mathrm{H}}^{n}} i_{ \pm}(X)=r(B)-i_{\mp}\left(A B^{*}\right) .
\end{gathered}
$$

Hence, the following hold.

(a) $A X=B$ has a nonsingular Hermitian solution if and only if $r(A)=r(B)$.

(b) $A X=B$ has a solution $X>0(X<0)$ if and only if $A B^{*} \geqslant 0$ and $r\left(A B^{*}\right)=r(A)\left(A B^{*} \leqslant 0\right.$ and $\left.r\left(A B^{*}\right)=r(A)\right)$.

(c) $A X=B$ has a solution $X \geqslant 0(X \leqslant 0)$ if and only if $A B^{*} \geqslant 0$ and $r\left(A B^{*}\right)=r(B)\left(A B^{*} \leqslant 0\right.$ and $\left.r\left(A B^{*}\right)=r(B)\right)$.

(d) The rank of the Hermitian solution of $A X=B$ is invariant $\Leftrightarrow$ the positive index of inertia of the Hermitian solution of $A X=B$ is invariant $\Leftrightarrow$ the negative index of inertia of the Hermitian solution of $A X=B$ is invariant $\Leftrightarrow r\left(A B^{*}\right)=r(A)+r(B)-n$.

Finally, we rewrite the matrix equation $A X=B$ as

$$
\left[A_{1}, A_{2}\right]\left[\begin{array}{ll}
X_{1} & X_{2} \\
X_{2}^{*} & X_{3}
\end{array}\right]=\left[B_{1}, B_{2}\right],
$$

where $A_{i} \in \mathbb{C}^{m \times n_{i}}, B_{i} \in \mathbb{C}^{m \times n_{i}}, X_{1} \in \mathbb{C}_{\mathrm{H}}^{n_{1}}, X_{2} \in \mathbb{C}^{n_{1} \times n_{2}}, X_{3} \in \mathbb{C}_{\mathrm{H}}^{n_{2}}$ for $i=1,2$ and $n_{1}+n_{2}=n$. Note that the unknown submatrices in (4.48) can be written as

$$
X_{1}=P_{1} X P_{1}^{*}, \quad X_{2}=P_{1} X P_{2}^{*}, \quad X_{3}=P_{2} X P_{2}^{*},
$$


where $P_{1}=\left[I_{n_{1}}, 0\right]$ and $P_{2}=\left[0, I_{n_{2}}\right]$. We next find the extremal ranks and inertias of the submatrices $X_{1}$ and $X_{3}$ in a Hermitian solution of (4.48). For convenience, let

$$
\begin{aligned}
& \mathcal{T}_{1}=\left\{X_{1} \in \mathbb{C}_{\mathrm{H}}^{n_{1}} \mid X_{1}=P_{1} X P_{1}^{*}, A X=B, X \in \mathbb{C}_{\mathrm{H}}^{n}\right\}, \\
& \mathcal{T}_{3}=\left\{X_{3} \in \mathbb{C}_{\mathrm{H}}^{n_{2}} \mid X_{3}=P_{2} X P_{2}^{*}, A X=B, X \in \mathbb{C}_{\mathrm{H}}^{n}\right\} .
\end{aligned}
$$

Applying Theorem 4.1 to (4.50) and (4.51) gives the following results. The details of the proof are omitted.

Theorem 4.6. Assume that matrix equation in (4.48) has a Hermitian solution, and let $\mathcal{T}_{1}$ and $\mathcal{T}_{3}$ be of the forms in (4.50) and (4.51). Then, the global maximal and minimal ranks and inertias of the Hermitian matrices in $\mathcal{T}_{1}$ and $\mathcal{T}_{3}$ are given by

$$
\begin{aligned}
\max _{X_{1} \in \mathcal{T}_{1}} r\left(X_{1}\right) & =n_{1}+r\left[A_{2}, B_{1}\right]-r(A), \\
\min _{X_{1} \in \mathcal{T}_{1}} r\left(X_{1}\right) & =2 r\left[A_{2}, B_{1}\right]-r\left[\begin{array}{cc}
A B^{*} & A_{2} \\
A_{2}^{*} & 0
\end{array}\right], \\
\max _{X_{1} \in \mathcal{T}_{1}} i_{ \pm}\left(X_{1}\right) & =n_{1}+i_{ \pm}\left[\begin{array}{cc}
A B^{*} & A_{2} \\
A_{2}^{*} & 0
\end{array}\right]-r(A), \\
\min _{X_{1} \in \mathcal{T}_{1}} i_{ \pm}\left(X_{1}\right) & =r\left[A_{2}, B_{1}\right]-i_{\mp}\left[\begin{array}{cc}
A B^{*} & A_{2} \\
A_{2}^{*} & 0
\end{array}\right],
\end{aligned}
$$

and

$$
\begin{aligned}
\max _{X_{3} \in \mathcal{T}_{3}} r\left(X_{3}\right) & =n_{2}+r\left[A_{1}, B_{2}\right]-r(A), \\
\min _{X_{3} \in \mathcal{T}_{3}} r\left(X_{3}\right) & =2 r\left[A_{1}, B_{2}\right]-r\left[\begin{array}{cc}
A B^{*} & A_{1} \\
A_{1}^{*} & 0
\end{array}\right], \\
\max _{X_{3} \in \mathcal{T}_{3}} i_{ \pm}\left(X_{3}\right) & =n_{2}+i_{ \pm}\left[\begin{array}{cc}
A B^{*} & A_{1} \\
A_{1}^{*} & 0
\end{array}\right]-r(A), \\
\min _{X_{3} \in \mathcal{T}_{3}} i_{ \pm}\left(X_{3}\right) & =r\left[A_{1}, B_{2}\right]-i_{\mp}\left[\begin{array}{cc}
A B^{*} & A_{1} \\
A_{1}^{*} & 0
\end{array}\right] .
\end{aligned}
$$

Applying Lemma 2.1 to (4.52)-(4.55), we easily obtain the following algebraic properties of the submatrix $X_{1}$ in (4.48).

Corollary 4.7. Assume that matrix equation in (4.48) has a Hermitian solution. Then, the following hold.

(a) (4.48) has a Hermitian solution in which $X_{1}$ is nonsingular if and only if $r\left[A_{2}, B_{1}\right]=r(A)$.

(b) $X_{1}$ is nonsingular in all Hermitian solutions of (4.48) if and only if

$$
r\left[\begin{array}{cc}
A B^{*} & A_{2} \\
A_{2}^{*} & 0
\end{array}\right]=2 r\left[A_{2}, B_{1}\right]-n_{1} .
$$

(c) (4.48) has a Hermitian solution in which $X_{1}>0\left(X_{1}<0\right)$ if and only if

$$
i_{+}\left[\begin{array}{cc}
A B^{*} & A_{2} \\
A_{2}^{*} & 0
\end{array}\right]=r(A) \quad\left(i_{-}\left[\begin{array}{cc}
A B^{*} & A_{2} \\
A_{2}^{*} & 0
\end{array}\right]=r(A)\right) .
$$


(d) $X_{1}>0\left(X_{1}<0\right)$ in all Hermitian solutions of (4.48) if and only if

$i_{-}\left[\begin{array}{cc}A B^{*} & A_{2} \\ A_{2}^{*} & 0\end{array}\right]=r\left[A_{2}, B_{1}\right]-n_{1} \quad\left(i_{+}\left[\begin{array}{cc}A B^{*} & A_{2} \\ A_{2}^{*} & 0\end{array}\right]=r\left[A_{2}, B_{1}\right]-n_{1}\right)$.

(e) (4.48) has a Hermitian solution in which $X_{1} \geqslant 0\left(X_{1} \leqslant 0\right)$ if and only if

$$
i_{+}\left[\begin{array}{cc}
A B^{*} & A_{2} \\
A_{2}^{*} & 0
\end{array}\right]=r\left[A_{2}, B_{1}\right] \quad\left(i_{-}\left[\begin{array}{cc}
A B^{*} & A_{2} \\
A_{2}^{*} & 0
\end{array}\right]=r\left[A_{2}, B_{1}\right]\right) .
$$

(f) $X_{1} \geqslant 0\left(X_{1} \leqslant 0\right)$ in all Hermitian solutions of (4.48) if and only if

$$
i_{-}\left[\begin{array}{cc}
A B^{*} & A_{2} \\
A_{2}^{*} & 0
\end{array}\right]=r(A)-n_{1} \quad\left(i_{+}\left[\begin{array}{cc}
A B^{*} & A_{2} \\
A_{2}^{*} & 0
\end{array}\right]=r(A)-n_{1}\right) .
$$

(g) (4.48) has a Hermitian solution in which $X_{1}=0$ if and only if $\mathscr{R}\left(B_{1}\right) \subseteq$ $\mathscr{R}\left(A_{2}\right)$.

(h) $X_{1}=0$ in all Hermitian solutions of (4.48) if and only if $r\left[A_{2}, B_{1}\right]=$ $r(A)-n_{1}$.

(i) The rank of $X_{1}$ in the Hermitian solution of (4.48) is invariant $\Leftrightarrow$ the positive index of inertia of $X_{1}$ in the Hermitian solution of (4.48) is invariant $\Leftrightarrow$ the negative index of inertia of $X_{1}$ in the Hermitian solution of (4.48) is invariant $\Leftrightarrow r\left[\begin{array}{cc}A B^{*} & A_{2} \\ A_{2}^{*} & 0\end{array}\right]=r\left[A_{2}, B_{1}\right]+r(A)-n_{1}$.

\section{Conclusions}

In this paper, we studied the problems of maximizing and minimizing the rank and partial inertia of the Hermitian matrix-valued function $A_{1}-B_{1} X B_{1}^{*}$ when $X$ runs over the two matrix sets in (1.2) and (1.3), respectively, and obtained many symbolic formulas for calculating the maximal and minimal ranks and inertias of $A_{1}-B_{1} X B_{1}^{*}$ by using pure algebraic operations of matrices and their generalized inverses. As direct applications, we gave necessary and sufficient conditions for the existence of $X$ satisfying the matrix equations in (1.8) and (1.14), as well as the Löwner partial ordering matrix inequalities in (1.9) and (1.15). Although there is no general theory for establishing closed-form formulas for calculating (maximal and minimal) ranks and inertias of matrices, the results presented in the previous sections as well as those in References [13]-[16] and [27]-[39], etc. by the present author show that many problems on calculations and optimizations of ranks and inertias of matrices can be solved explicitly by using some pure algebraic operations of matrices, while the closed-form formulas obtained can be used to solve many fundamental problems in matrix theory, as mentioned in the beginning of this paper. This work seems extremely fruitful, while the results obtained in these papers are easy to understand within the scope of elementary linear algebra. This series of seminal researches show that for many basic or classic problems like solvability of matrix equations and matrix inequalities, we are still able to establish a variety of innovative results by some tricky algebraic methods.

Motivated by the fruitful work on ranks and inertias of matrices, lots of people did much parallel work in recent years on establishing formulas for calculating 
ranks and inertias of matrices, and many results obtained in their papers were overlapped with those in this paper and References [13]-[16] and [27]-[39], etc. In these cases, the originality of those overlapped results should belong to the comprehensive work in this paper, [13]-[16] and [27]-[39], etc. by the present author.

As a continuation, we mention some research problems on Hermitian matrixvalued functions and Hermitian matrix equations for further consideration:

(a) A challenging task is to give the closed-form of the general common Hermitian solution of $B_{2} X B_{2}^{*}=A_{2}$ and $B_{3} X B_{3}^{*}=A_{3}$ that satisfies $X>0$ $(\geqslant 0,<0, \leqslant 0)$, which is equivalent to solving the following Hermitian matrix inequalities

$X_{0}+V F_{B}+F_{B} V^{*}+F_{B_{2}} U F_{B_{3}}+F_{B_{3}} U^{*} F_{B_{2}}>0(\geqslant 0,<0, \leqslant 0)$.

Moreover, give the extremal ranks and inertias of the Hermitian matrixvalued function $A_{1}-B_{1} X B_{1}^{*}$ subject to $B_{2} X B_{2}^{*}=A_{2}$ and $B_{3} X B_{3}^{*}=A_{3}$ and $X \geqslant 0(X \leqslant 0)$.

(b) Derive possible closed-form formulas for calculating the extremal ranks and inertias of the Hermitian matrix-valued function $A_{1}-B_{1} X B_{1}^{*}$ subject to common Hermitian solutions of the $k-1$ consistent Hermitian matrix equations

$$
\left[B_{2} X B_{2}^{*}, \ldots, B_{k} X B_{k}^{*}\right]=\left[A_{2}, \ldots, A_{k}\right],
$$

and establish necessary and sufficient condition for the set of Hermitian matrix equations

$$
\left[B_{1} X B_{1}^{*}, \ldots, B_{k} X B_{k}^{*}\right]=\left[A_{1}, \ldots, A_{k}\right]
$$

to have a common Hermitian solution, as well as a common semi-definite solution, respectively.

(c) Derive possible closed-form formulas for calculating the extremal ranks and inertias of the Hermitian matrix-valued function $A_{1}-B_{1} X B_{1}^{*}$ subject to a Hermitian matrix inequality $B_{2} X B_{2}^{*} \geqslant A_{2}\left(B_{2} X B_{2}^{*} \leqslant A_{2}\right)$. In such a case, it is necessary to first give a closed-form formula of general Hermitian solution of $B_{2} X B_{2}^{*} \geqslant A_{2}\left(B_{2} X B_{2}^{*} \leqslant A_{2}\right)$.

(d) Derive possible closed-form formulas for calculating the the extremal ranks and inertias of $A_{1}-B_{1} X B_{1}^{*}$ subject to $B_{2} X=A_{2}$ and $X \geqslant 0(X \leqslant 0)$.

Acknowledgements. The author is grateful to an anonymous referee for helpful comments and suggestions on this paper. This work was supported by National Natural Science Foundation of China (Grant No. 11271384).

\section{REFERENCES}

1. E. Candes and B. Recht, Exact matrix completion via convex optimization, Found. Comput. Math. 9 (2009), 717-772.

2. M. Fazel, H. Hindi and S. Boyd, A Rank minimization heuristic with application to minimum order system approximation, Proceedings of the 2001 American Control Conference, 4734-4739, 2001.

3. M. Fazel, H. Hindi and S. Boyd, Rank minimization and applications in system theory, Proceedings of the 2004 American Control Conference, 3273-3278, 2004. 
4. J.F. Geelen, Maximum rank matrix completion, Linear Algebra Appl. 288 (1999), 211-217.

5. J. Groß, $A$ note on the general Hermitian solution to $A X A^{*}=B$, Bull. Malays. Math. Soc. (2) 21 (1998), 57-62.

6. N.J.A. Harvey, D.R. Karger and S. Yekhanin, The complexity of matrix completion, Proceedings of the Seventeenth Annual ACM-SIAM Symposium on Discrete Algorithm, Association for Computing Machinery, New York, pp. 1103-1111, 2006.

7. T.M. Hoang and T. Thierauf, The complexity of the inertia, Lecture Notes in Comput. Sci. 2556, Springer, pp. 206-217, 2002.

8. T.M. Hoang and T. Thierauf, The complexity of the inertia and some closure properties of GapL, Proceedings of the Twentieth Annual IEEE Conference on Computational Complexity, pp. 28-37, 2005.

9. C.G. Khatri and S.K. Mitra, Hermitian and nonnegative definite solutions of linear matrix equations, SIAM J. Appl. Math. 31 (1976), 579-585.

10. Y. Kim and M. Mesbahi, On the rank minimization problem, Proceedings of the 2004 American Control Conference, Boston, pp. 2015-2020, 2004.

11. M. Laurent, Matrix completion problems, Encyclopedia of Optimization (C.A. Floudas and P.M. Pardalos, eds.), Vol. III, Kluwer, pp. 221-229, 2001.

12. X. Liu and J. Rong, On Hermitian nonnegative-definite solutions to matrix equations, Math. Notes 85 (2009), 453-457.

13. Y. Liu and Y. Tian, More on extremal ranks of the matrix expressions $A-B X \pm X^{*} B^{*}$ with statistical applications, Numer. Linear Algebra Appl. 15 (2008), 307-325.

14. Y. Liu and Y. Tian, Extremal ranks of submatrices in an Hermitian solution to the matrix equation $A X A^{*}=B$ with applications, J. Appl. Math. Comput. 32 (2010), 289-301.

15. Y. Liu and Y. Tian, A simultaneous decomposition of a matrix triplet with applications, Numer. Linear Algebra Appl. 18 (2011), 69-85.

16. Y. Liu and Y. Tian, Max-min problems on the ranks and inertias of the matrix expressions $A-B X C \pm(B X C)^{*}$ with applications, J. Optim. Theory Appl. 148 (2011), 593-622.

17. Y. Liu and Y. Tian, Hermitian-type of singular value decomposition for a pair of matrices and its applications, Numer. Linear Algebra Appl. 20 (2013), 60-73.

18. Y. Liu, Y. Tian and Y. Takane, Ranks of Hermitian and skew-Hermitian solutions to the matrix equation $A X A^{*}=B$, Linear Algebra Appl. 431 (2009), 2359-2372.

19. G. Marsaglia and G.P.H. Styan, Equalities and inequalities for ranks of matrices, Linear Multilinear Algebra 2 (1974), 269-292.

20. M. Mahajan and J. Sarma, On the complexity of matrix rank and rigidity, Lecture Notes in Comput. Sci. 4649, Springer, pp. 269-280, 2007.

21. M. Mesbahi, On the rank minimization problem and its control applications, Systems Control Letters 33 (1998), 31-36.

22. M. Mesbahi and G.P. Papavassilopoulos, Solving a class of rank minimization problems via semi-definite programs, with applications to the fixed order output feedback synthesis, Proceedings of the American Control Conference, Albuquerque, New Mexico, pp. 77-80, 1997.

23. B.K. Natarajan, Sparse approximate solutions to linear systems, SIAM J. Comput. 24 (1995), 227-234.

24. A.B. Özgüler and N. Akar, A common solution to a pair of linear matrix equations over a principal ideal domain, Linear Algebra Appl. 144 (1991), 85-99.

25. B. Recht, M. Fazel and P.A. Parrilo, Guaranteed minimum rank solutions to linear matrix equations via nuclear norm minimization, SIAM Review 52 (2010), 471-501.

26. Y. Tian, Solvability of two linear matrix equations, Linear Multilinear Algebra 48 (2000), 123-147.

27. Y. Tian, Equalities and inequalities for inertias of Hermitian matrices with applications, Linear Algebra Appl. 433 (2010), 263-296.

28. Y. Tian, Rank and inertia of submatrices of the Moore-Penrose inverse of a Hermitian matrix, Electron. J. Linear Algebra 20 (2010), 226-240. 
29. Y. Tian, Completing block Hermitian matrices with maximal and minimal ranks and inertias, Electron. J. Linear Algebra 21 (2010), 124-141.

30. Y. Tian, Expansion formulas for the inertias of Hermitian matrix polynomials and matrix pencils of orthogonal projectors, J. Math. Anal. Appl. 376 (2011), 162-186.

31. Y. Tian, Maximization and minimization of the rank and inertia of the Hermitian matrix expression $A-B X-(B X)^{*}$ with applications, Linear Algebra Appl. 434 (2011), 2109-2139.

32. Y. Tian, Solutions to 18 constrained optimization problems on the rank and inertia of the linear matrix function $A+B X B^{*}$, Math. Comput. Modelling 55 (2012), 955-968.

33. Y. Tian, On additive decompositions of the Hermitian solutions of the matrix equation $A X A^{*}=B$, Mediterr. J. Math. 9 (2012), 47-60.

34. Y. Tian, Solving optimization problems on ranks and inertias of some constrained nonlinear matrix functions via an algebraic linearization method, Nonlinear Anal. 75 (2012), 717-734.

35. Y. Tian, Formulas for calculating the extremum ranks and inertias of a four-term quadratic matrix-valued function and their applications, Linear Algebra Appl. 437 (2012), 835-859.

36. Y. Tian, Least-squares solutions and least-rank solutions of the matrix equation $A X A^{*}=B$ and their relations, Numer. Linear Algebra Appl., in press.

37. Y. Tian, Equalities and inequalities for Hermitian solutions and Hermitian definite solutions of the two matrix equations $A X=B$ and $A X A^{*}=B$, Aequationes Math., in press.

38. Y. Tian and Y. Li, Distributions of eigenvalues and inertias of some block Hermitian matrices consisting of orthogonal projectors, Linear Multilinear Algebra 60 (2012), 1027-1069.

39. Y. Tian and Y. Liu, Extremal ranks of some symmetric matrix expressions with applications, SIAM J. Matrix Anal. Appl. 28 (2006), 890-905.

40. X. Zhang, The general common Hermitian Nonnegative-definite solution to the matrix equations $A X A^{*}=B$ and $C X C^{*}=D$, Linear Multilinear Algebra 52 (2004), 49-60.

41. X. Zhang, The general common Hermitian nonnegative-definite solution to the matrix equations $A X A^{*}=B B^{*}$ and $C X C^{*}=D D^{*}$ with applications in statistics, J. Multivariate Anal. 93 (2005), 257-266.

Cema, Central University of Finance and Economics, Beijing 100081, China. E-mail address: yongge.tian@gmail.com 